\title{
The proof-theoretic strength of Ramsey's theorem for pairs and two colors
}

\author{
Ludovic Patey ${ }^{1}$ and Keita Yokoyama ${ }^{2}$ \\ $1_{\text {ludovic. patey@computability.fr }}$ \\ ${ }^{2}$ y-keita@jaist.ac.jp \\ March 17, 2016 (first version) \\ March 15, 2018 (revised version)
}

\begin{abstract}
Ramsey's theorem for $n$-tuples and $k$-colors $\left(\mathrm{RT}_{k}^{n}\right)$ asserts that every $k$-coloring of $[\mathbb{N}]^{n}$ admits an infinite monochromatic subset. We study the proof-theoretic strength of Ramsey's theorem for pairs and two colors, namely, the set of its $\Pi_{1}^{0}$ consequences, and show that $\mathrm{RT}_{2}^{2}$ is $\Pi_{3}^{0}$ conservative over $\mathrm{I} \Sigma_{1}^{0}$. This strengthens the proof of Chong, Slaman and Yang that $\mathrm{RT}_{2}^{2}$ does not imply $\mathrm{I} \Sigma_{2}^{0}$, and shows that $\mathrm{RT}_{2}^{2}$ is finitistically reducible, in the sense of Simpson's partial realization of Hilbert's Program. Moreover, we develop general tools to simplify the proofs of $\Pi_{3}^{0}$-conservation theorems.
\end{abstract}

Key words: Reverse Mathematics, Ramsey's theorem, proof-theoretic strength

MSC (2010): Primary 03B30, 03F35, 05D10, Secondary 03H15, 03C62, 03D80

\section{Introduction}

Ramsey's theorem for $n$-tuples and $k$-colors $\left(\mathrm{RT}_{k}^{n}\right)$ asserts that every $k$-coloring of $[\mathbb{N}]^{n}$ admits an infinite monochromatic subset. Ramsey's theorem is probably the most famous theorem of Ramsey's theory, and plays a central role in combinatorics and graph theory (see, e.g., [28, 25]) with numerous applications in mathematics and computer science, among which functional analysis [2] automata theory [53], or termination analysis [54]. An important aspect of Ramsey's theorem is its definable class of fast-growing functions. Erdös [21] showed that the (diagonal) Ramsey number has an exponential growth rate. Actually, Ramsey's theorem defines much faster-growing functions, which is studied by Ketonen and Solovay [37], among others. The growth rate of these functions have important applications, since it provides upper bounds to combinatorial questions

The authors are grateful to Theodore A. Slaman and Emanuele Frittaion for useful comments and discussions. We also thank Leszek Kołodziejczyk for pointing out a flaw in Definitions 2.5 and 3.1.

Ludovic Patey is funded by the John Templeton Foundation ('Structure and Randomness in the Theory of Computation' project). The opinions expressed in this publication are those of the author(s) and do not necessarily reflect the views of the John Templeton Foundation.

Keita Yokoyama is partially supported by JSPS KAKENHI (grant numbers 16K17640 and 15H03634) and JSPS Core-to-Core Program (A. Advanced Research Networks).

Part of this work in this paper was done during the Dagstuhl Seminar 15392 "Measuring the Complexity of Computational Content: Weihrauch Reducibility and Reverse Analysis". 
from various fields. This type of question is heavily related to proof theory, and with their language, the question is formalized as follows:

What is the class of functions whose existence is provable (with an appropriate base system) from Ramsey's theorem?

For example, the Ramsey number function belongs to this class since the existence of the Ramsey number $R(n, k)$ is guaranteed by Ramsey's theorem. In fact, this class of functions decides the so-called "proof-theoretic strength" of Ramsey's theorem.

Ramsey's theorem also plays a very important role in reverse mathematics as it is one of the main examples of theorems escaping the Big Five phenomenon (see Section 1.2). Reverse mathematics is a general program that classifies theorems by two different measures, namely, by their computability-theoretic strength and by their proof-theoretic strength. As it happens, consequences of Ramsey's theorem are notoriously hard to study in reverse mathematics, and therefore received a lot of attention from the reverse mathematics community. Especially, determining the strength of Ramsey's theorem for pairs $\left(\mathrm{RT}_{2}^{2}\right)$ is always a central topic in the study of reverse mathematics. This study yielded series of seminal papers [34, 55, 13, 15] introducing both new computability-theoretic and proof-theoretic techniques. (See Section 1.2 for more details of its computability-theoretic strength.)

In this paper, we mainly focus on the proof-theoretic strength of Ramsey's theorem for pairs. By the proof-theoretic strength of a theory $T$ we mean the set of $\Pi_{1}^{0}$ sentences which are provable in $T$, or the proof-theoretic ordinal of $T$ which is decided by the class of ( $\Sigma_{1}^{0}$-definable) functions whose totality are proved in $T$. In fact, we will give the exact proof-theoretic strength of $\mathrm{RT}_{2}^{2}$ by proving that $\mathrm{RT}_{2}^{2}+\mathrm{WKL}_{0}$ is a $\Pi_{3}^{0}$-conservative extension of $\mathrm{I} \Sigma_{1}^{0}$ (Theorem 7.4), where $\mathrm{WKL_{0 }}$ stands for weak König's lemma and I $\Sigma_{n}^{0}$ is the $\Sigma_{n}^{0}$-induction scheme. This answers the longstanding open question of determining the $\Pi_{2}^{0}$-consequences of $\mathrm{RT}_{2}^{2}$ or the consistency strength of $\mathrm{RT}_{2}^{2}$, posed, e.g., in Seetapun and Slaman [55, Question 4.4] Cholak, Jockusch and Slaman [13, Question 13.2] Chong and Yang [17] (see Corollaries 7.5 and 7.6). For this, we use a hybrid of forcing construction, indicator arguments, and proof-theoretic techniques, and develop general tools simplifying conservation results (see Theorems 3.1, 3.4 and 6.1). See Section 1.3 for the various studies of the proof-theoretic strength of $\mathrm{RT}_{2}^{2}$. Deciding the proof-theoretic strength of $\mathrm{RT}_{2}^{2}$ is also an important problem from a philosophical point of view. In the sense of Simpson's partial realization [56] of Hilbert's Program, one would conclude that $\mathrm{RT}_{2}^{2}$ is finitistically reducible (see Section 1.5).

\subsection{Reverse mathematics}

Reverse mathematics is a vast foundational program that seeks to determine which set existence axioms are needed to prove theorems from "ordinary" mathematics. It uses the framework of subsystems of second-order arithmetic. Indeed, Friedman [?] realized that a large majority of theorems admitted a natural formulation in the language of second-order arithmetic. The base theory $\mathrm{RCA}_{0}$, standing for Recursive Comprehension Axiom, contains the basic axioms for firstorder arithmetic (axioms of discrete ordered semi-ring) together with the $\Delta_{1}^{0}$-comprehension scheme and the $\Sigma_{1}^{0}$-induction scheme. $\mathrm{RCA}_{0}$ can be thought of as capturing computable mathematics.

Since then, thousands of theorems have been studied within the framework of reverse mathematics. A surprising phenomenon emerged from the early years of reverse mathematics: Most theorems studied require very weak axioms. Moreover, many of them happen to be equivalent to 
one of five main sets of axioms, that are referred to as the Big Five, namely, RCA $A_{0}$, weak König's lemma $\left(\mathrm{WKL}_{0}\right)$, the arithmetic comprehension axiom $\left(\mathrm{ACA}_{0}\right)$, arithmetical transfinite recursion $\left(A T_{0}\right)$, and $\Pi_{1}^{1}$-comprehension axiom $\left(\Pi_{1}^{1}-C A_{0}\right)$. See Simpson [57] for an extensive study of the Big Five and mathematics within them. In this paper, we shall consider exclusively theorems which are provable in $\mathrm{ACA}_{0}$. See Hirschfeldt [31] for a gentle introduction to the reverse mathematics below $\mathrm{ACA}_{0}$.

\subsection{Ramsey's theorem and its consequences}

Ramsey theory is a branch of mathematics studying the conditions under which some structure appears among a sufficiently large collection of objects. In the past two decades, Ramsey theory emerged as one of the most important topics in reverse mathematics. This theory provides a large class of theorems escaping the Big Five phenomenon, and whose strength is notoriously hard to gauge. Perhaps the most famous such theorem is Ramsey's theorem.

Definition 1.1 (Ramsey's theorem). A subset $H$ of $\mathbb{N}$ is homogeneous for a coloring $f:[\mathbb{N}]^{n} \rightarrow k$ (or $f$-homogeneous) if all the $n$-tuples over $H$ are given the same color by $f . \mathrm{RT}_{k}^{n}$ is the statement "Every coloring $f:[\mathbb{N}]^{n} \rightarrow k$ has an infinite $f$-homogeneous set".

Jockusch [34] conducted a computational analysis of Ramsey's theorem, later formalized by Simpson [57] within the framework of reverse mathematics. Whenever $n \geq 3$, Ramsey's theorem for $n$-tuples happens to be equivalent to $\mathrm{ACA}_{0}$. The status of Ramsey's theorem for pairs was open for decades, until Seetapun and Slaman [55] proved that $\mathrm{RT}_{2}^{2}$ is strictly weaker than $\mathrm{ACA}_{0}$ over RCA . Cholak, Jockusch and Slaman [13] extensively studied Ramsey's theorem for pairs. On a computability-theoretic perspective, every computable instance of $\mathrm{RT}_{k}^{n}$ admits a $\Pi_{n}^{0}$ solution, while there exists a computable instance of $\mathrm{RT}_{2}^{n}$ with no $\Sigma_{n}^{0}$ solution [34]. Ramsey's theorem for pairs is computationally weak in that it does not imply the existence of PA degrees [44], or any fixed incomputable set [55].

In order to better understand the logical strength of $\mathrm{RT}_{2}^{2}$, Bovykin and Weiermann [10] decomposed Ramsey's theorem for pairs into the Erdős-Moser theorem and the ascending descending sequence principle. The Erdős-Moser is a statement from graph theory.

Definition 1.2 (Erdős-Moser theorem). A tournament $T$ is an irreflexive binary relation such that for all $x, y \in \mathbb{N}$ with $x \neq y$, exactly one of $T(x, y)$ or $T(y, x)$ holds. A tournament $T$ is transitive if the corresponding relation $T$ is transitive in the usual sense. EM is the statement "Every infinite tournament $T$ has an infinite transitive subtournament."

Definition 1.3 (Ascending descending sequence). Given a linear order (i.e., a transitive tournament) $<_{L}$ on $\mathbb{N}$, an ascending (descending) sequence is a set $S$ such that for every $x<_{\mathbb{N}} y \in S$, $x<_{L} y\left(x>_{L} y\right)$. ADS is the statement "Every infinite linear order admits an infinite ascending or descending sequence".

The Erdős-Moser theorem provides together with the ascending descending principle an alternative decomposition of Ramsey's theorem for pairs. Indeed, every coloring $f:[\mathbb{N}]^{2} \rightarrow 2$ can be seen as a tournament $R$ such that $R(x, y)$ holds if $x<y$ and $f(x, y)=1$, or $x>y$ and $f(y, x)=0$. Then, EM is saying "Every coloring $f:[\mathbb{N}]^{n} \rightarrow k$ has an infinite transitive subcoloring" and ADS is saying "Every transitive coloring $f:[\mathbb{N}]^{n} \rightarrow k$ has an infinite $f$-homogeneous set". (In what follows, we always consider EM and ADS as these forms.) We therefore obtain the following equivalence. 
Theorem 1.1 (Hirschfeldt and Shore [32], Bovykin and Weiermann [10]). RCA $\vdash \mathrm{RT}_{2}^{2} \leftrightarrow$ ADS + EM.

The ascending descending sequence has been introduced by Hirschfeldt and Shore [32]. They proved that ADS is strictly weaker than Ramsey's theorem for pairs. On the other hand, Lerman, Solomon and Towsner [43] proved that the Erdős-Moser theorem is strictly weaker than $\mathrm{RT}_{2}^{2}$. For technical purposes, we shall consider a statement equivalent to the ascending descending principle. Pseudo Ramsey's theorem for pairs has been introduced by Murakami, Yamazaki and the second author [47] to study a factorization theorem from automata theory.

Definition 1.4 (Pseudo Ramsey's theorem for pairs). A set $H$ is pseudo-homogeneous for a coloring $f:[\mathbb{N}]^{2} \rightarrow k$ if there is a color $c<k$ such that every pair $\{x, y\} \in[H]^{2}$ are the endpoints of a finite sequence $x_{0}<x_{1}<\cdots<x_{n}$ such that $f\left(x_{i}, x_{i+1}\right)=c$ for each $i<n$. $\operatorname{psRT}_{k}^{2}$ is the statement "Every coloring $f:[\mathbb{N}]^{2} \rightarrow k$ has an infinite $f$-pseudo-homogeneous set".

In particular, if $f:[\mathbb{N}]^{2} \rightarrow 2$ is a transitive coloring, then any set $H$ pseudo-homogeneous for $f$ is already homogeneous for $f$. Thus, $\mathrm{RCA}_{0}+\mathrm{psRT}_{2}^{2}$ implies ADS (see [47]). The first author [52] and Steila (see [59]) independently proved the reverse implication, namely, $\mathrm{RCA}_{0}+\mathrm{ADS}$ implies $\mathrm{psRT}_{2}^{2}$.

\subsection{Proof strength and conservation results}

In the study of reverse mathematics, deciding the first-order or proof-theoretic strength of axioms and mathematical principles is one of the main topics. This is usually analyze through the conservation theorems. Especially, the conservation result for weak König's lemma always plays the central role as a large part of mathematics can be proven within $\mathrm{WKL}_{0}$ (see Simpson [57]). The following theorems show that one can use weak König's lemma almost freely to seek for first-order consequences.

Theorem 1.2 (Friedman[23], see [57]). WKL 0 is a $\Pi_{2}^{0}$-conservative extension of PRA.

Theorem 1.3 (Harrington, see [57]). WKL $\mathrm{W}_{0}$ is a $\Pi_{1}^{1}$-conservative extension of $\mathrm{I} \Sigma_{1}^{0}$.

The $\Sigma_{2}^{0}$-bounding principle $\left(\mathrm{B} \Sigma_{2}^{0}\right)$ informally asserts that a finite union of finite sets is finite. Many mathematical reasonings make an essential use of $\mathrm{B} \Sigma_{2}^{0}$ and in particular $\mathrm{RT}_{2}^{2}$ implies $\mathrm{B} \Sigma_{2}^{0}$. The strength of the $\Sigma_{2}^{0}$-bounding principle is therefore important for the study of combinatorial principles.

Theorem 1.4 (Hájek[26]). $W K L_{0}+\mathrm{B} \Sigma_{2}^{0}$ is a $\Pi_{1}^{1}$-conservative extension of $\mathrm{B} \Sigma_{2}^{0}$.

Thankfully, $\mathrm{B} \Sigma_{2}^{0}$ can be freely used for a restricted class of formulas. Let $\tilde{\Pi}_{3}^{0}$ be a class of formulas of the form $\forall X \varphi(X)$ where $\varphi$ is a $\Pi_{3}^{0}$-formula. The following is a parameterized version of the Parsons, Paris and Friedman conservation theorem.

Theorem 1.5 (see, e.g., [12] or [36]). $\mathrm{B} \Sigma_{2}^{0}$ is a $\tilde{\Pi}_{3}^{0}$-conservative extension of $\mathrm{I} \Sigma_{1}^{0}$.

Note that the above four conservation theorems are frequently used in this paper, and so we shall not mention them explicitely.

About the first-order/proof-theoretic strength of Ramsey's theorem for pairs, there are long series of studies by various people and various methods. Hirst [33] showed that $\mathrm{RT}_{2}^{2}$ implies the $\Sigma_{2}^{0}$-bounding principle $\left(\mathrm{B} \Sigma_{2}^{0}\right)$. On the other hand, Cholak, Jockusch and Slaman [13] showed that $\mathrm{WKL}_{0}+\mathrm{RT}_{2}^{2}+\mathrm{I} \Sigma_{2}^{0}$ is a $\Pi_{1}^{1}$-conservative extension of $\mathrm{I} \Sigma_{2}^{0}$. Thus, the first-order strength of 
Ramsey's theorem for pairs and two colors is in between $\mathrm{B} \Sigma_{2}^{0}$ and $\mathrm{I} \Sigma_{2}^{0}$. After this work, many advanced studies are done to investigate the first-order strength of Ramsey's theorem and related combinatorial principles. One of the most important methods for these studies consists in adapting computability-theoretic techniques for combinatorial principles. By this method, Chong, Slaman and Yang [16] showed that two weaker combinatorial principles, namely, the ascending descending sequence (ADS) and the chain antichain principle (CAC), introduced by Shore and Hirschfeldt [32], are $\Pi_{1}^{1}$-conservative over $\mathrm{B} \Sigma_{2}^{0}$. In [15], they showed that $\mathrm{WKL}_{0}+\mathrm{SRT}_{2}^{2}$ does not imply $\mathrm{I} \Sigma_{2}^{0}$, and they improved the result and proved that $\mathrm{RT}_{2}^{2}$ does not imply $\mathrm{I} \Sigma_{2}^{0}$ in [14]. More recently, Chong, Kreuzer and Yang [unpublished] showed that $\mathrm{WKL}_{0}+\mathrm{SRT}_{2}^{2}$ is $\Pi_{3}^{0}$-conservative over $\mathrm{RCA}_{0}+\mathrm{WF}\left(\omega^{\omega}\right)$, where $\mathrm{WF}\left(\omega^{\omega}\right)$ asserts the well-foundedness of $\omega^{\omega}$.

Besides the computability-theoretic techniques, many other significant approaches can be found in the literature. Kohlenbach and Kreuzer [42] and Kreuzer [41] characterized the $\Pi_{2}^{0}$-parts of $\mathrm{RT}_{2}^{2}$ and CAC with several different settings by proof-theoretic approaches. Bovykin and Weiermann [10] and the second author [65] showed that indicators defined by Paris's density notion can approach the proof-theoretic strength of various versions of Ramsey's theorem, and by a similar method, the second author [66] also showed that $\mathrm{RT}_{k}^{n}+\mathrm{WKL}_{0}^{*}$ is fairly weak and is a $\Pi_{2}^{0}$-conservative extension of $\mathrm{RCA}_{0}^{*}$, where $\mathrm{RCA}_{0}^{*}$ is $\mathrm{RCA}_{0}$ with only $\Sigma_{0}^{0}$-induction and the exponentiation. There are also many studies of the proof-theoretic strength of Ramsey's theorem by using ordinal analysis, by Kotlarski, Weiermann, et al. [63, 39, 7, 8, 9, 58].

Moreover, the study of proof-theoretic strength of Ramsey's theorem for pairs has a solid connection to computer science. Especially, in the field of termination analysis, Podelski and Rybalchenko [54] introduced a new method to verify the termination of programs by using Ramsey's theorem for pairs, and based on this method, many termination verifiers are invented. On the other hand, as we can see in Buchholz [11], it is known that proof theory can provide an upper bound for termination proofs since the termination statement is always described by a $\Pi_{2}^{0}$-formula. In fact, the termination theorem argued in [54] is essentially equivalent to a weaker version of Ramsey's theorem for pairs, and the proof-theoretic strength of Ramsey's theorem can give a general upper bound for all of those types of termination proofs. See [59].

\subsection{Second-order structures of arithmetic and their cuts}

A structure for the language of second-order arithmetic $\mathcal{L}_{2}$ is a pair $(M, S)$ where $M=\left(M,+_{M},{ }_{M}\right.$, $0_{M}, 1_{M},<_{M}$ ) is a structure for the language of first-order (Peano) arithmetic $\mathcal{L}_{\mathrm{PA}}$, and $S$ is a subset of the power set of $M$.

Definition 1.5 (Cut). Given a structure $M$ of the first-order arithmetic, a substructure $I \subseteq M$ is said to be a $c u t$ of $M$ (abbreviated $\left.\left(I \subseteq_{e} M\right)\right)$ if $I=\left\{a \in M \mid \exists b \in I\left(a<_{M} b\right)\right\}$.

Here, the standard first-order structure $\omega$ can be considered as the smallest cut of any first-order structure. Given a structure $(M, S)$, a cut $I \subseteq_{e} M$ induces the second-order structure $(I, S\lceil I)$, where $S\lceil I:=\{X \cap I \mid X \in S\}$. We sometimes consider $S$ as a family of unary predicates on $M$ and identify $(M, S)$ as an $\mathcal{L}_{\mathrm{PA}} \cup S$-structure. Accordingly, $(I, S\lceil I)$ can be considered as an $\mathcal{L}_{\mathrm{PA}} \cup S$-substructure of $(M, S)$. Note that $S \mid I$ may then be a multiset on $I$, but this is harmless without second-order equality. In this sense, one can easily check that $\left(I, S\lceil I)\right.$ is a $\Sigma_{0}^{0}$-elementary substructure of $(M, S)$ if $I$ is closed under $+_{M}$ and $\cdot_{M}$.

We write $\operatorname{Cod}(M)$ for the collection of all $M$-finite subsets, i.e., subsets of $M$ canonically coded by elements in $M$ (as the usual binary expansion). Given some cut $I \subseteq_{e} M$, we write $\operatorname{Cod}(M / I)$ 
for $\operatorname{Cod}(M)\left\lceil I\right.$. If $I \subsetneq M$, then $\operatorname{Cod}(M / I)=S\left\lceil I\right.$ for any $S \subseteq \mathcal{P}(M)$ such that $(M, S) \models \mathrm{RCA}_{0}$, thus $(I, S\lceil I)$ only depends on $M$ and $I$.

A cut $I \subseteq_{e} M$ is said to be semi-regular if $I \cap X$ is bounded for any $X \in \operatorname{Cod}(M)$ such that $|X| \in I$, where $|X|$ denotes the cardinality of $X$ in $M$. A semi-regular cut is one of the central notions in the study of cuts, especially with the connection to second-order structures, since it characterizes the models of $\mathrm{WKL}_{0}$. We will use the following theorem throughout this paper without mentioning it explicitly.

Theorem 1.6 (see, e.g., Theorems 7.1.5, 7.1.7 of [38]). Let I be a cut of a first-order structure $M$. Then, $I$ is semi-regular if and only if $(I, \operatorname{Cod}(M / I)) \models \mathrm{WKL}_{0}$.

Bounding principles are also characterized by cuts with some elementarity condition. In this paper, we will use the following characterization.

Theorem 1.7 (Proposition 3 of Clote [18], see also Paris and Kirby [49]). Let $n \geq 1$. Let $(M, S)$ be a model of $\mathrm{I} \Sigma_{n-1}^{0}$, and let $I$ be a cut of $M$ such that $\left(I, S\lceil I)\right.$ be a $\Sigma_{n}^{0}$-elementary substructure of $(M, S)$. Then, $(I, S \mid I) \models \mathrm{B} \Sigma_{n+1}^{0}$.

\subsection{Hilbert's program and finitistic reductionism}

During the early 20th century, mathematics went through a serious foundational crisis, with the discovery of various paradoxes and inconsistencies. Some great mathematicians such as Kronecker, Poincaré and Brouwer challenged the validity of infinitistic reasoning. Hilbert [29] proposed a three-step program to answer those criticisms. First, he suggested to identify the finitistic part of mathematics, then to axiomatize infinite reasoning, and eventually to give a finitistically correct consistency proof of this axiomatic system. However, his program was nipped in the bud by Gödel's incompleteness theorems [24].

In 1986, Simpson [56] proposed a formal interpretation of Hilbert's program by taking primitive recursive arithmetic (PRA) as the base system for capturing finitistic reasoning. This choice was convincingly justified by Tait [60]. Simpson took second-order arithmetic $\left(Z_{2}\right)$ as the big system capturing infinitistic reasoning, based on the work of Hilbert and Bernays [30]. In this setting, finitistic reductionism can be interpreted as proving that $Z_{2}$ is $\Pi_{1}^{0}$-conservative over PRA. By Gödel incompleteness theorem, this cannot be the case. However, Simpson proposed to consider $\Pi_{1}^{0}$-conservation of subsystems of second-order arithmetic over PRA as a partial realization of Hilbert's program. He illustrated his words with weak König's lemma (WKL) which was proven by Friedman to be $\Pi_{2}^{0}$-conservative over PRA (Theorem 1.2). In this paper, we contribute to Hilbert's program by showing that $\mathrm{WKL}_{0}+\mathrm{RT}_{2}^{2}$ is $\Pi_{2}^{0}$-conservative over PRA, and therefore that $\mathrm{RT}_{2}^{2}$ is finitistically reducible. Moreover, we develop general tools to simplify the proofs of $\Pi_{3}^{0}$ conservation theorems, and thereby contribute to the simplification of the realization of Hilbert's program.

\subsection{Notation}

In order to avoid confusion between the theory and the meta-theory, we shall use $\omega$ to denote the set of (standard) natural numbers, and $\mathbb{N}$ to denote the sets of natural numbers inside the system. Accordingly, we shall write $\omega$ for the ordinal $\omega$ in the system. We write $[a, b]_{\mathbb{N}},(a, b)_{\mathbb{N}},(a, \infty)_{\mathbb{N}}, \ldots$ for intervals of natural numbers, e.g., $(a, b]_{\mathbb{N}}=\{x \in \mathbb{N} \mid a<x \leq b\}$. Given a set $X$ and some $n \in \mathbb{N}$, $[X]^{n}$ is the collection of all sets of size $n .[X]^{<\mathbb{N}}$ is the union $\bigcup_{n}[X]^{n}$. 
We use $\Pi_{n}, \Sigma_{n}, \Delta_{n}$ to denote first-order formulas without set parameters, whereas $\Pi_{n}^{0}, \Sigma_{n}^{0}$, $\Delta_{n}^{0}$ are second-order formulas, i.e., with set parameters. A $\tilde{\Pi}_{n}^{0}$-formula is a second-order formula of the form $(\forall X) \varphi(X)$ where $\varphi$ is a $\Pi_{n}^{0}$-formula.

Given two sets $A, B, A \oplus B=\{2 x \mid x \in A\} \cup\{2 x+1 \mid x \in B\}, A \subseteq_{\text {fin }} B$ means that $A$ is a finite subset of $B$, and $A \subseteq^{*} B$ means that the set $A$ is included, up to finite changes, in $B$. We write $A<B$ for the formula $(\forall x \in A)(\forall y \in B) x<y$. Whenever $A=\{x\}$, we shall simply write $x<B$ for $A<B$. A set $X$ can be seen as an infinite join $X=\bigoplus_{i} X_{i}$, where $x \in X_{i}$ iff $\langle i, x\rangle \in X$. We then write $X[i]$ for $X_{i}$. Given a set $X$ or a string $\sigma$ and some integer $m \in \omega$, we write $X\lceil m$ for the initial segment of $X$ (resp. $\sigma$ ) of length $m$.

\section{7 $\quad$ Structure of this paper}

The main target of this paper is the following conservation theorem.

Theorem. $\mathrm{WKL}_{0}+\mathrm{RT}_{2}^{2}$ is a $\tilde{\Pi}_{3}^{0}$-conservative extension of $\mathrm{I} \Sigma_{1}^{0}$.

We will prove this in the following way.

In Sections 2 and 3, we will explain that $\tilde{\Pi}_{3}^{0}$-consequences of Ramsey's theorem and its variations are characterized by some largeness notions of finite sets. We will introduce largeness notion for $\Gamma$, where $\Gamma$ is any of $\mathrm{RT}_{2}^{2}$, $\mathrm{psRT}_{2}^{2}$ (which is equivalent to ADS), and EM. Roughly speaking, giving a bound for largeness for $\Gamma$ within $\mathrm{I} \Sigma_{1}^{0}$ provides $\tilde{\Pi}_{3}^{0}$-conservation for $\mathrm{WK} \mathrm{L}_{0}+\Gamma$ over $\mathrm{I} \Sigma_{1}^{0}$ (Theorem 3.1).

According to the decomposition of $\mathrm{RT}_{2}^{2}$ into $\mathrm{ADS}$ and $\mathrm{EM}$ and the amalgamation theorem (Theorem 3.6), the conservation for $\mathrm{RT}_{2}^{2}$ can be decomposed into the conservation for ADS and the conservation for EM. In Section 4, we give a bound for the largeness notion for $\operatorname{psRT}_{2}^{2}$ (Lemma 4.4) by using the Ketonen/Solovay theorem. It actually provides the conservation result for ADS (Corollary 4.5).

It is rather complicated to give a bound for the largeness notion for EM. For this, we will introduce a new combinatorial principle called the grouping principle. We mainly focus on the grouping principle for pairs and two colors $\mathrm{GP}_{2}^{2}$. Section 5 is devoted to the reverse mathematical study of $\mathrm{GP}_{2}^{2}$, especially from the view point of computability theory. In Section 6, we will prove a conservation theorem for $\mathrm{GP}_{2}^{2}$ (Theorem 6.5). For this, we will modify the construction of a low solution for the stable version of $\mathrm{GP}_{2}^{2}$ (Theorem 5.2) presented in the previous section.

In Section 7, we give a bound for the largeness notion for EM (Lemma 7.2) by using a finite version of the grouping principle, which is a consequence of $\mathrm{GP}_{2}^{2}$. It provides the conservation result for EM (Theorem 7.3). Then, combining this with the conservation result in Section 4 by the amalgamation theorem, we obtain the main theorem.

The main theorem can be formalized within $\mathrm{WKL}_{0}$, and that leads to the consistency equivalence of $\mathrm{I} \Sigma_{1}^{0}$ and $\mathrm{RT}_{2}^{2}$. This is argued in Section 8.

\section{$2 \quad$ Largeness}

A family of finite sets of natural numbers $L \subseteq[\mathbb{N}]^{<\mathbb{N}}$ is said to be a largeness notion if any infinite set has a finite subset in $\mathrm{L}$ and $\mathrm{L}$ is closed under supersets. A finite set $X \in[\mathbb{N}]^{<\mathbb{N}}$ is said to be L-large if $X \in \mathrm{L}$. A (possibly largeness) notion $\mathrm{L} \subseteq[\mathbb{N}]^{<\mathbb{N}}$ is said to be regular if for any L-large set $F$, any finite set $G \subseteq_{\text {fin }} \mathbb{N}$ for which there exists an order-preserving injection $h: F \rightarrow G$ such that $(\forall x \in F) h(x) \leq x$, then $G \in \mathrm{L}$. A $\Delta_{0}$-definable notion $\mathrm{L} \subseteq[\mathbb{N}]^{<\mathbb{N}}$ is said to be a (regular) 
largeness notion provably in I $\Sigma_{1}^{0}$ if $\mathrm{I} \Sigma_{1}^{0} \vdash$ "L is a (regular) largeness notion". The idea of a largeness notion is introduced in Aczel [1] (it is called 'density' in [1]). In this paper, we shall mainly consider regular largeness notions provably in $\mathrm{I} \Sigma_{1}^{0}$.

Example 2.1. The family $\mathrm{L}_{\omega}=\left\{X \subseteq_{\text {fin }} \mathbb{N}:|X|>\min X\right\}$ is a regular largeness notion provably in I $\Sigma_{1}^{0}$.

The notion of largeness enjoys a property similar to the pigeonhole principle, as states the following lemma.

Lemma $2.1\left(\mathrm{WKL}_{0}+\mathrm{B} \Sigma_{2}^{0}\right)$. For any largeness notion $\mathrm{L}$, for any infinite set $X$ and for any $k, N_{0} \in \mathbb{N}$, there exists $N_{1} \in \mathbb{N}$ such that for any partition $X \cap\left[N_{0}, N_{1}\right]_{\mathbb{N}}=X_{0} \sqcup \cdots \sqcup X_{k-1}$, there exists an $\mathrm{L}$-large set $F$ such that $F \subseteq X_{i}$ for some $i<k$.

Since $W K L_{0}+B \Sigma_{2}^{0}$ is a $\tilde{\Pi}_{3}^{0}$-conservative extension of $\mathrm{I} \Sigma_{1}^{0}$, this lemma for a $\Delta_{1}$-definable largeness notion provably in $\mathrm{I} \Sigma_{1}^{0}$ is provable in $\mathrm{I} \Sigma_{1}^{0}$.

Proof. By B $\Sigma_{2}^{0}$, for any partition $X \cap\left[N_{0}, \infty\right)_{\mathbb{N}}=X_{0} \sqcup \cdots \sqcup X_{k-1}$, one of the $X_{i}$ 's is infinite and thus it contains an L-large subset. Thus, a bound for such an L-large set can be obtained by the usual compactness argument which is available within $\mathrm{WKL}_{0}$.

Remark 2.2. Note that the use of $\mathrm{B} \Sigma_{2}^{0}$ in the previous lemma is essential. If $\mathrm{B} \Sigma_{2}^{0}$ fails, there exists a partition $X=X_{0} \sqcup \cdots \sqcup X_{k-1}$ such that each of the $X_{i}$ 's is finite. Then, $\mathrm{L}=\left\{F \in[\mathbb{N}]^{<\mathbb{N}} \mid\right.$ $\left.\forall i<k\left(F \nsubseteq X_{i}\right)\right\}$ is a largeness notion failing the lemma. The use of $\mathrm{WKL}_{0}$ is not essential since the argument can be formalized within RWKL $+\mathrm{B} \Sigma_{2}^{0}$, where RWKL denotes the Ramsey-type weak König's lemma introduced by Flood [22].

\section{$2.1 \quad \alpha$-largeness}

From now on, we fix a primitive recursive notation for ordinals below $\epsilon_{0}$. In this paper, we actually use ordinals of the form $\alpha=\sum_{i<k} \omega^{n_{i}}<\omega^{\omega}$ where $n_{i} \in \mathbb{N}$ and $n_{0} \geq \cdots \geq n_{k-1}$. (We write 1 for $\omega^{0}$, and $\omega^{n} \cdot k$ for $\sum_{i<k} \omega^{n}$.) For a given $\alpha<\omega^{\omega}$ and $m \in \mathbb{N}$, define $0[m]=0, \alpha[m]=\beta$ if $\alpha=\beta+1$ and $\alpha[m]=\beta+\omega^{n-1} \cdot m$ if $\alpha=\beta+\omega^{n}$ for some $n \geq 1$.

Definition $2.2\left(\mathrm{I} \Sigma_{1}^{0}\right)$. Let $\alpha<\omega^{\omega}$. A set $X=\left\{x_{0}<\cdots<x_{\ell-1}\right\} \subseteq_{\text {fin }} \mathbb{N}$ is said to be $\alpha$-large if $\alpha\left[x_{0}\right] \ldots\left[x_{\ell-1}\right]=0$. In other words, any finite set is 0-large, and $X$ is said to be $\alpha$-large if

- $X \backslash\{\min X\}$ is $\beta$-large if $\alpha=\beta+1$,

- $X \backslash\{\min X\}$ is $\left(\beta+\omega^{n-1} \cdot \min X\right)$-large if $\alpha=\beta+\omega^{n}$.

We let $\mathrm{L}_{\alpha}=\left\{X \subseteq_{\text {fin }} \mathbb{N} \mid X\right.$ is $\alpha$-large $\}$.

In particular, a set $X$ is $m$-large iff $|X| \geq m$ and $\omega$-large iff $|X|>\min X$. See [27] for the general definition of $\alpha$-largeness. One can easily see that if $X \subseteq Y$ for some $\alpha$-large set $X$ and some finite set $Y$, then $Y$ is $\alpha$-large.

We say that $X$ is $\alpha$-small if it is not $\alpha$-large. The following basic combinatorics have been proven in [27, Theorem II.3.21] in their full generality.

Lemma $2.3\left(\mathrm{I} \Sigma_{1}^{0}\right)$. Fix any $k, n \in \mathbb{N}$.

(i) A set $X$ is $\omega^{n} \cdot k$-large if and only if it is a union of some $\omega^{n}$-large finite sets $X_{0}<\cdots<X_{k-1}$. 
(ii) A set $X$ is $\omega^{n} \cdot k$-small if it is a union of $\omega^{n}$-small finite sets $X_{0}<\cdots<X_{k-1}$.

In particular, $\{k\} \cup X_{0} \cup \cdots \cup X_{k-1}$ is $\omega^{n+1}$-large if each of $X_{i}$ is $\omega^{n}$-large and $k<X_{0}<\cdots<$ $X_{k-1}$. Similarly, if $\{k\} \cup X_{0} \cup \cdots \cup X_{k-1}$ is $\omega^{n+1}$-large and $k<X_{0}<\cdots<X_{k-1}$, then one of $X_{i}$ 's is $\omega^{n}$-large.

The following theorem corresponds to the well-known fact that the proof-theoretic ordinal of $\mathrm{I} \Sigma_{1}^{0}$ is $\omega^{\omega}$.

Theorem 2.4. For any $n \in \omega, \mathrm{I} \Sigma_{1}^{0}$ proves that $\mathrm{L}_{\omega^{n}}$ is a regular largeness notion.

Proof. One can easily check the regularity within $\mathrm{I} \Sigma_{1}^{0}$. We will see that $\mathrm{I} \Sigma_{1}^{0} \vdash$ "any infinite set contains an $\omega^{n}$-large subset" by (external) induction. The case $n=0$ is trivial. We show the case $n=k+1$. Within $\mathrm{I} \Sigma_{1}^{0}$, let an infinite set $X$ be given. Then, by the induction hypothesis and $\Sigma_{1}^{0}$ induction, one can find $\min X$-many $\omega^{k}$-large sets $F_{i} \subseteq X$ such that $\min X<F_{0}<\cdots<F_{\min } X-1$. By Lemma 2.3 and the discussion below it, $\{\min X\} \cup F_{0} \cup \cdots \cup F_{\min X-1}$ is $\omega^{k+1}$-large.

\subsection{Largeness for Ramsey-like statements}

Many Ramsey-type theorems studied in reverse mathematics are statements of the form "For every coloring $f:[\mathbb{N}]^{n} \rightarrow k$, there is an infinite set $H$ satisfying some structural properties". The most notable example is Ramsey's theorem, which asserts for every coloring $f:[\mathbb{N}]^{n} \rightarrow k$ the existence of an infinite $f$-homogeneous set. These statements can be seen as mathematical problems, whose instances are coloring, and whose solutions are the sets satisfying the desired structural properties.

Definition 2.3. A Ramsey-like- $\Pi_{2}^{1}$-formula is a $\Pi_{2}^{1}$-formula of the form

$$
\left(\forall f:[\mathbb{N}]^{n} \rightarrow k\right)(\exists Y)(Y \text { is infinite } \wedge \Psi(f, Y))
$$

where $n, k \in \omega$ and $\Psi(f, Y)$ is of the form $\left(\forall G \subseteq_{\text {fin }} Y\right) \Psi_{0}\left(f \uparrow\left[[0, \max G]_{\mathbb{N}}\right]^{n}, G\right)$ such that $\Psi_{0}$ is a $\Delta_{0}^{0}$-formula.

In particular, $\mathrm{RT}_{k}^{n}$ is a Ramsey-like- $\Pi_{2}^{1}$-statement where $\Psi(f, Y)$ is the formula " $Y$ is homogeneous for $f$ ". Similarly, psRT $_{k}^{2}$ and EM are Ramsey-like- $\Pi_{2}^{1}$ statements. On the other hand, $\mathrm{SRT}_{2}^{2}$ is not a Ramsey-like- $\Pi_{2}^{1}$-statement. However, $\mathrm{SRT}_{2}^{2}$ is equivalent to the Ramsey-like- $\Pi_{2}^{1}$-formula saying "for any 2-coloring $f$ on $[\mathbb{N}]^{2}$, there exists an infinite set $Y$ such that $Y$ is homogeneous for $f$ or there exists $a<\min Y$ witnessing the non-stability of $f$, that is, such that for any $x, y \in Y$ there exist $b, c \in[x, y)_{\mathbb{N}}$ such that $f(a, b) \neq f(a, c)$ ". Although the definition of a Ramsey-like- $\Pi_{2}^{1}$-formula seems very restrictive, we can show that it entails a much larger class of $\Pi_{2}^{1}$-statements.

Definition 2.4. A restricted- $\Pi_{2}^{1}$-formula is a $\Pi_{2}^{1}$-formula of the form $\forall X \exists Y \Theta(X, Y)$ where $\Theta$ is a $\Sigma_{3}^{0}$-formula.

Proposition 2.5. For any restricted- $\Pi_{2}^{1}$-formula $\forall X \exists Y \Theta(X, Y)$, there exists a Ramsey-like- $\Pi_{2}^{1}$ formula $\forall X \exists Z(Z$ is infinite $\wedge \Psi(X, Z))$ such that

$$
\mathrm{WKL}_{0} \vdash \forall X(\exists Y \Theta(X, Y) \leftrightarrow \exists Z(Z \text { is infinite } \wedge \Psi(X, Z))) \text {. }
$$

(Here, $X$ is considered as a function $X:[\mathbb{N}]^{1} \rightarrow 2$ in the definition of Ramsey-like- $\Pi_{2}^{1}$-formula.)

Proof. We work within $\mathrm{WKL}_{0}$. Let $\forall X \exists Y \Theta(X, Y)$ be a restricted- $\Pi_{2}^{1}$-formula. Without loss of generality, one can write $\Theta(X, Y) \equiv \forall n \exists m \theta\left(X\left\lceil m, Y\lceil m, n, m)\right.\right.$ where $\theta$ is $\Sigma_{0}^{0}$ since existential 
number quantifier can be replaced with an existential set quantifier. Define a formula $\Psi(X, Z)$ as follows:

$$
\Psi(X, Z) \equiv \forall F \subseteq_{\text {fin }} Z\left(F \neq \emptyset \rightarrow\left(\exists \sigma \in 2^{\max F}\right)(\forall n<|F|)(\exists m<\max F) \theta(X\lceil m, \sigma\lceil m, n, m)) .\right.
$$

We now show that $\exists Y \Theta(X, Y) \leftrightarrow \exists Z(Z$ is infinite $\wedge \Psi(X, Z))$.

To show the left to right implication, take $Y \subseteq \mathbb{N}$ such that $\forall n \exists m \theta(X\lceil m, Y\lceil m, n, m)$. Define an infinite increasing sequence $\left\langle z_{i} \mid i \in \mathbb{N}\right\rangle$ as $z_{0}=\min \left\{m+1 \mid \theta\left(X\lceil m, Y\lceil m, 0, m)\}\right.\right.$, and $z_{i+1}=$ $\min \left\{m>z_{i} \mid \theta\left(X\lceil m, Y\lceil m, i+1, m)\}\right.\right.$. Let $Z=\left\{z_{i} \mid i \in \mathbb{N}\right\}$, then we have $Z$ is infinite $\wedge \Psi(X, Z)$ (given a non-empty set $F \subseteq_{\text {fin }} Z$, set $\sigma=Y\lceil\max F$ ).

To show the right to left implication, take $Z \subseteq \mathbb{N}$ such that $Z$ is infinite $\wedge \Psi(X, Z)$. Define a tree $T$ as

$$
T=\left\{\sigma \in 2^{<\mathbb{N}} \mid \forall F \subseteq Z \cap[0,|\sigma|]_{\mathbb{N}}(F \neq \emptyset \rightarrow(\forall n<|F|)(\exists m<\max F) \theta(X\lceil m, \sigma\lceil m, k, n, m))\} .\right.
$$

Then, since $\Psi(X, Z)$ holds, the tree $T$ is infinite. By $\mathrm{WKL}_{0}$, take $Y \in[T]$. One can check that $\forall n \exists m \theta(X\lceil m, Y\lceil m, n, m)$ since $Z$ is infinite.

Definition $2.5\left(\mathrm{I} \Sigma_{1}^{0}\right)$. Fix an ordinal $\alpha<\omega^{\omega}$ and a Ramsey-like- $\prod_{2}^{1}$-statement $\Gamma \equiv\left(\forall f:[\mathbb{N}]^{n} \rightarrow\right.$ $k)(\exists Y)(Y$ is infinite $\wedge \Psi(f, Y))$. A set $Z \subseteq_{\text {fin }} \mathbb{N}$ is said to be $\alpha$-large $(\Gamma)$ if for any $f:\left[[0,|Z|)_{\mathbb{N}}\right]^{n} \rightarrow k$, there is an $\alpha$-large set $Y \subseteq Z$ such that $\Psi\left(f, p_{Z}(Y)\right)$ holds, where $p_{Z}$ is the unique order preserving bijection from $Z$ to $[0,|Z|)_{\mathbb{N}}$.

By the definition of a Ramsey-like- $\Pi_{2}^{1}$-formula, if $Z^{\prime} \supseteq Z$ and $Z$ is $\alpha$-large( $(\Gamma)$, then $Z^{\prime}$ is $\alpha$ large $(\Gamma)$. For the usual Ramsey type statements we consider $\left(\mathrm{RT}_{k}^{n}, \mathrm{psRT}_{k}^{2}, \mathrm{EM}, \ldots\right)$, we usually identify a function $f:\left[[0,|Z|)_{\mathbb{N}}\right]^{n} \rightarrow k$ with $f \circ\left(p_{Z}\right)^{n}:[Z]^{n} \rightarrow k$ and just discuss on $[Z]^{n}$.

Example 2.6. A set $Z$ is $\alpha$-large $\left(\mathrm{RT}_{k}^{n}\right)$ if for every coloring $f:[Z]^{n} \rightarrow k$, there is an $\alpha$-large $f$-homogeneous set $Y \subseteq Z$.

Note that $\alpha$-largeness $(\Gamma)$ for $\Gamma \in\left\{\mathrm{RT}_{k}^{n}, \mathrm{psRT}_{k}^{2}, \mathrm{EM}\right\}$ are all $\Delta_{0}$-definable notions, and $\mathrm{I} \Sigma_{1}^{0}$ proves that they are all regular. However, it is not obvious within $\mathrm{I} \Sigma_{1}^{0}$ that they are all largeness notions. Actually, showing within $\mathrm{I} \Sigma_{1}^{0}$ that $\alpha$-largeness $\left(\mathrm{RT}_{2}^{2}\right)$ is a largeness notion is the key to know the $\Pi_{3}^{0}$-part of $\mathrm{RT}_{2}^{2}$. Our approach is to measure the size of $\alpha$-large $(\Gamma)$ sets by comparing them with $\alpha$-large sets. The following classical theorem is fundamental for this purpose. (It is not hard to check that the proof works within $\mathrm{I} \Sigma_{1}^{0}$.)

Theorem 2.6 (Ketonen and Solovay[37, Section 6]). Let $k \in \omega$. The following is provable within $\mathrm{I} \Sigma_{1}^{0}$. If a finite set $X$ is $\omega^{k+4}$-large and $\min X>3$, then $X$ is $\omega$-large $\left(\mathrm{RT}_{k}^{2}\right)$.

\section{Density and $\tilde{\Pi}_{3}^{0}$-conservation}

The goal of this section is to prove the following theorem.

Theorem 3.1 (Conservation through largeness). Let $\Gamma$ be a Ramsey-like- $\Pi_{2}^{1}$-statement. If $\omega^{k}$ large $(\Gamma)$ ness is a largeness notion provably in $\mathrm{I} \Sigma_{1}^{0}$ for any $k \in \omega$, then, $\mathrm{WKL}_{0}+\Gamma$ is a $\tilde{\Pi}_{3}^{0}$ conservative extension of $\mathrm{I} \Sigma_{1}^{0}$.

For this, we will introduce an iterated version of a largeness notion which is called "density". This notion is introduced by Paris in [48]. 
Definition 3.1 ( $\mathrm{I} \Sigma_{1}^{0}$, Density notion). Fix a Ramsey-like $\Pi_{2}^{1}$-statement

$$
\Gamma=\left(\forall f:[\mathbb{N}]^{n} \rightarrow k\right)(\exists Y)(Y \text { is infinite } \wedge \Psi(f, Y))
$$

We define the notion of $m$-density $(\Gamma)$ of a finite set $Z \subseteq \mathbb{N}$ inductively as follows. First, a set $Z$ is 0 -dense $(\Gamma)$ if it is $\omega$-large and $\min Z>1$. Assuming the notion of $m$-density $(\Gamma)$ is defined, a set $Z$ is $(m+1)$-dense $(\Gamma)$ if

- for any $f:\left[[0,|Z|)_{\mathbb{N}}\right]^{n} \rightarrow k$, there is an $m$-dense $(\Gamma)$ set $Y \subseteq Z$ such that $\Psi\left(f, p_{Z}(Y)\right)$ holds, where $p_{Z}$ is the unique order preserving bijection from $Z$ to $[0,|Z|)_{\mathbb{N}}$, and,

- for any partition $Z_{0} \sqcup \cdots \sqcup Z_{\ell-1}=Z$ such that $\ell \leq Z_{0}<\cdots<Z_{\ell-1}$, one of $Z_{i}$ 's is $m$-dense $(\Gamma)$.

Note that there exists a $\Delta_{0}$-formula $\theta(m, Z)$ saying that " $Z$ is $m$-dense $(\Gamma)$." (Here, we always assume $\min Z>1$ to avoid technical annoyances of the second condition.)

In case $\Gamma$ is $\operatorname{psRT}_{k}^{2}$ or $\mathrm{RT}_{k}^{n}$ for some $n, k \geq 2$, the second condition is implied from the first condition as follows: for a given partition $Z_{0} \sqcup \cdots \sqcup Z_{\ell-1}=Z$, set $f(x, y)=1$ if $x, y \in Z_{i}$ for some $i<\ell$ and $f(x, y)=0$ otherwise, then, $f$ is a transitive coloring and any $\omega$-large homogeneous set $H \subseteq Z$ is included in some $Z_{i}$ 's. (For more precise explanations, see [48] or [10].) On the other hand, the density notion for EM without the second condition does not work well (see [10]).

Definition 3.2 (Paris-Harrington principle for density). Let $\Gamma$ be a Ramsey-like- $\Pi_{2}^{1}$-statement. Then, the statement $m-\widetilde{\mathrm{PH}}(\Gamma)$ asserts that for any $X_{0} \subseteq \mathbb{N}$, if $X_{0}$ is infinite then there exists an $m$-dense $(\Gamma)$ set $X$ such that $X \subseteq_{\text {fin }} X_{0}$.

The density notion for $\Gamma$ provides a cut to be a model of $W K L_{0}+\Gamma$.

Lemma 3.2. Let $\Gamma$ be a Ramsey-like- $\Pi_{2}^{1}$-statement. Given a countable nonstandard model $M$ of $\mathrm{I} \Sigma_{1}$ and an $M$-finite set $Z \subseteq M$ which is a-dense $(\Gamma)$ for some $a \in M \backslash \omega$, then there exists an initial segment $I$ of $M$ such that $(I, \operatorname{Cod}(M / I))=\mathrm{WKL}_{0}+\Gamma$ and $I \cap Z$ is infinite in $I$.

Proof. Let $\Gamma$ be a Ramsey-like- $\Pi_{2}^{1}$-statement of the form

$$
\left(\forall f:[\mathbb{N}]^{n} \rightarrow k\right)(\exists Y)(Y \text { is infinite } \wedge \Psi(f, Y))
$$

where $n, k \in \omega$ and $\Psi$ is of the form in Definition 2.3. Let $M \models \mathrm{I} \Sigma_{1}$ be a countable nonstandard model, and $Z \subseteq M$ be $M$-finite set which is $a$-dense( $\Gamma)$ for some $a \in M \backslash \omega$. Let $\left\{E_{i}\right\}_{i \in \omega}$ be an enumeration of all $M$-finite sets such that any $M$-finite set appears infinitely many times, and $\left\{f_{i}\right\}_{i \in \omega}$ be an enumeration of all $M$-finite functions from $\left[[0,|Z|)_{\mathbb{N}}\right]^{n}$ to $k$.

In the following, we will construct an $\omega$-length sequence of $M$-finite sets $Z=Z_{0} \supseteq Z_{1} \supseteq \ldots$ so that for each $i \in \omega, Z_{i}$ is $(a-i)$-dense $(\Gamma), \min Z_{i}<\min Z_{i+3}, \Psi\left(f_{i} \uparrow\left[\left[0, \mid Z_{3 i}\right)_{\mathbb{N}}\right]^{n}, p_{Z_{3 i}}\left(Z_{3 i+1}\right)\right)$, and $\left(\min Z_{3 i+2}, \max Z_{3 i+2}\right)_{\mathbb{N}} \cap E_{i}=\emptyset$ if $\left|E_{i}\right|<\min Z_{3 i+1}$.

At the stage $s=3 i$, let $Z_{3 i}$ and $f_{i}$ be given. Then, one can find $Z_{3 i+1} \subseteq Z_{3 i}$ which is $(a-3 i-1)$-dense $(\Gamma)$ such that $\Psi\left(f_{i} \uparrow\left[\left[0,\left|Z_{3 i}\right|\right)_{\mathbb{N}}\right]^{n}, p_{Z_{3 i}}\left(Z_{3 i+1}\right)\right)$ by the definition of $\operatorname{density}(\Gamma)$.

At the stage $s=3 i+1$, let $Z_{3 i+1}$ and $E_{i}$ be given. If $\min Z_{3 i+1} \leq\left|E_{i}\right|$, let $Z_{3 i+2}=Z_{3 i+1}$. If $\min Z_{3 i+1}>\left|E_{i}\right|$, let $E_{i}=\left\{e_{0}, \ldots, e_{l-1}\right\}$ where $e_{0}<e_{1}<\cdots<e_{l-1}$, and put $W^{0}=Z_{3 i+1} \cap\left[0, e_{0}\right)_{\mathbb{N}}$, $W^{j}=Z_{3 i+1} \cap\left[e_{j-1}, e_{j}\right)_{\mathbb{N}}$ for $1 \leq j<l$, and $W^{l}=Z_{3 i+1} \cap\left[e_{l-1}, \infty\right)_{\mathbb{N}}$. Then, $Z_{3 i+1}=W^{0} \sqcup \cdots \sqcup W^{l}$, thus one of $W_{j}$ 's is $(a-3 i-2)$-dense $(\Gamma)$. Put $Z_{3 i+2}$ to be such $W_{j}$.

At the stage $s=3 i+2$, Put $Z_{3 i+3}=Z_{3 i+2} \backslash\left\{\min Z_{3 i+2}\right\}$. 
Now, let $I=\sup \left\{\min Z_{i} \mid i \in \omega\right\} \subseteq_{e} M$. By the construction of the steps $s=3 i+1, I$ is a semi-regular cut, thus $(I, \operatorname{Cod}(M / I)) \models \mathrm{WKL}_{0}$. By the construction of the steps $s=3 i+2, Z_{i} \cap I$ is infinite in $I$ for any $i \in \omega$. To check that $(I, \operatorname{Cod}(M / I)) \models \Gamma$, let $f:[I]^{n} \rightarrow k \in \operatorname{Cod}(M / I)$. Then, there exists $f_{i}$ such that $f=f_{i} \cap I$. By the construction, $M \models \Psi\left(f_{i} \uparrow\left\lceil\left[0,\left|Z_{3 i}\right|\right)_{\mathbb{N}}\right]^{n}, p_{Z_{3 i}}\left(Z_{3 i+1}\right)\right)$ holds, thus we have $(I, \operatorname{Cod}(M / I)) \models \Psi\left(f, p_{Z_{3 i}}\left(Z_{3 i+1}\right) \cap I\right)$. Moreover, since $Z_{3 i+1} \cap I$ is infinite in $I$ and $p_{Z_{3 i}}(x) \leq x$ for any $x \in Z, p_{Z_{3 i}}\left(Z_{3 i+1}\right) \cap I$ is also infinite in $I$.

Now the density version of Paris-Harrington principle characterize the $\tilde{\Pi}_{3}^{0}$-part of Ramsey-like statements. The following theorem is a generalization of [10, Theorem 1]

Theorem 3.3. Let $\Gamma$ be a Ramsey-like- $\Pi_{2}^{1}$-statement. Then, $\mathrm{WKL}_{0}+\Gamma$ is a $\tilde{\Pi}_{3}^{0}$-conservative extension of $\mathrm{RCA}_{0}+\{m-\widetilde{\mathrm{PH}}(\Gamma) \mid m \in \omega\}$.

Proof. By the usual compactness argument, one can easily check that $\mathrm{WKL}_{0}+\Gamma$ implies $m-\widetilde{\mathrm{PH}}(\Gamma)$ for any $m \in \omega$. Thus, $\mathrm{WKL}_{0}+\Gamma$ is an extension of $\mathrm{RCA}_{0}+\{m-\widetilde{\mathrm{PH}}(\Gamma) \mid m \in \omega\}$.

To see that it is a $\tilde{\Pi}_{3}^{0}$-conservative extension, let $\varphi_{0} \equiv \forall X \forall x \exists y \forall z \varphi(X[z], x, y, z)$ be a $\tilde{\Pi}_{3}^{0}$ sentence which is not provable in $\mathrm{RCA}_{0}+\{m-\widetilde{\mathrm{PH}}(\Gamma) \mid m \in \omega\}$, where $\varphi$ is $\Sigma_{0}^{0}$. Take a countable nonstandard model $(M, S) \models \mathrm{RCA}_{0}+\{m-\widetilde{\mathrm{PH}}(\Gamma) \mid m \in \omega\}+\neg \varphi_{0}$. Then, there exist $A \in S$ and $a \in M$ such that $(M, S) \models \forall y \exists z \neg \varphi(A[z], a, y, z)$. In $(M, S)$, define a sequence $\left\langle x_{i} \mid i \in M\right\rangle$ so that $x_{0}=a$ and $x_{i+1}=\min \left\{x>x_{i} \mid \forall y<x_{i} \exists z<x \neg \varphi(A[z], a, y, z)\right\}$. By recursive comprehension in $(M, S)$, put $X=\left\{x_{i} \mid i \in M\right\} \in S$. Then, $X$ is infinite in $(M, S)$. By $m-\widetilde{\mathrm{PH}}(\Gamma)$ for $m \in \omega$, there exist $m$-dense $(\Gamma)$ finite subsets of $X$ for any $m \in \omega$. Thus, by overspill for $\Sigma_{1}^{0}$-statement, there exists an $m$-dense( $\Gamma$ ) finite subset $Z$ of $X$ for some $m \in M \backslash \omega$. Now, by Lemma 3.2, there exists $I \subsetneq e M$ such that $(I, \operatorname{Cod}(M / I)) \models \mathrm{WKL}_{0}+\Gamma$ and $Z \cap I$ is infinite in $I$. Note that $\operatorname{Cod}(M / I)=\{W \cap I \mid W$ is $M$-finite $\}=\{W \cap I \mid W \in S\}$. Since $Z \subseteq X, a \leq \min Z \in I$ and for any $w, w^{\prime} \in Z \cap I$ such that $w<w^{\prime},(I, \operatorname{Cod}(M / I)) \models \forall y<w \exists z<w^{\prime} \neg \varphi(A \cap I[z], a, y, z)$. Since $Z \cap I$ is unbounded in $I$, we have $(I, \operatorname{Cod}(M / I)) \models \forall y \exists z \neg \varphi(A \cap I[z], a, y, z)$, which means $(I, \operatorname{Cod}(M / I)) \models \neg \varphi_{0}$. Thus, $\mathrm{WKL}_{0}+\Gamma$ does not prove $\varphi_{0}$.

The density notion actually captures some finite consequences of Ramsey-like-statements as follows.

Theorem 3.4. Let $\Gamma$ be a Ramsey-like- $\Pi_{2}^{1}$-statement, and let $\psi(x, y, F)$ be a $\Delta_{0}$-formula with exactly the displayed free variables. Assume that

$$
\mathrm{WKL}_{0}+\Gamma \vdash \forall x \forall X\left(X \text { is infinite } \rightarrow \exists F \subseteq_{\text {fin }} X \exists y \psi(x, y, F)\right) .
$$

Then, there exists $n \in \omega$ such that

$$
\mathrm{I} \Sigma_{1} \vdash \forall x \forall Z \subseteq_{\text {fin }}(x, \infty)_{\mathbb{N}}(Z \text { is } n \text {-dense }(\Gamma) \rightarrow \exists F \subseteq Z \exists y<\max Z \psi(x, y, F)) .
$$

Proof. Assume that $\mathrm{I} \Sigma_{1}^{0} \forall \forall x \forall Z \subseteq_{\text {fin }}(x, \infty)_{\mathbb{N}}(Z$ is $n$-dense $(\Gamma) \rightarrow \exists F \subseteq Z \exists y<\max Z \psi(x, y, F))$ for any $n \in \omega$. Then, there exists a countable model $M \models I \Sigma_{1}+\left\{\exists x \exists Z \subseteq_{\text {fin }}(x, \infty)_{\mathbb{N}}(Z\right.$ is $n$ dense $(\Gamma) \wedge \forall F \subseteq Z \forall y<\max Z \neg \psi(x, y, F)) \mid n \in \omega\}$ such that $M \neq \omega$. By overspill, there exists $a \in M \backslash \omega$ such that $M \models \exists x \exists Z \subseteq_{\text {fin }}(x, \infty)_{\mathbb{N}}(Z$ is $a$-dense $(\Gamma) \wedge \forall F \subseteq Z \forall y<\max Z \neg \psi(x, y, F))$, thus there exist $c \in M$ and an $M$-finite set $Z \subseteq M$ with $\min Z>c$ such that $Z$ is $a$-dense $(\Gamma)$ and $\forall F \subseteq Z \forall y<\max Z \neg \psi(c, y, F)$.

Now, by Lemma 3.2, there exists $I \subsetneq e$ such that $(I, \operatorname{Cod}(M / I)) \models \mathrm{WKL}_{0}+\Gamma$ and $Z \cap I$ is infinite in $I$. Note that $c \in I$. Thus, we have $(I, \operatorname{Cod}(M / I)) \models\left(Z \cap I\right.$ is infinite $\wedge \forall F \subseteq_{\text {fin }} Z \cap$ $I \forall y \neg \psi(c, y, F)))$. This contradicts to $\mathrm{WKL}_{0}+\Gamma \vdash \forall x \forall X\left(X\right.$ is infinite $\left.\rightarrow \exists F \subseteq_{\text {fin }} X \exists y \psi(x, y, F)\right)$. 
The argument we used in Lemma 3.2, Theorems 3.3 and 3.4 is a generalization of (a special case of) the well-known indicator argument (see, e.g., [48, 36]). Actually, by Theorem 3.3 and Proposition 2.5, one can characterize the $\tilde{\Pi}_{3}^{0}$-part of any restricted- $\Pi_{2}^{1}$-statement, as same as the usual indicator arguments captures $\Pi_{2}^{0}$-parts. In general, one can replace the second condition and the initial condition for 0-density in Definition 3.1 with suitable indicator conditions for a base system $T$, then the partial conservation for $T+\Gamma$ over $T+\{m-\widetilde{\mathrm{PH}}(\Gamma) \mid m \in \omega\}$ holds, and one can even consider the $\tilde{\Pi}_{4}^{0}$-part in some cases. See $[65,66]$.

The following corollary of the previous theorem plays a key role in this paper.

Corollary 3.5 (Generalized Parsons theorem). Let $\psi(F)$ be a $\Sigma_{1}$-formula with exactly the displayed free variables. Assume that

$$
\mathrm{I} \Sigma_{1}^{0} \vdash \forall X \subseteq \mathbb{N}(X \text { is infinite } \rightarrow \exists F \subseteq \text { fin } X \psi(F)) .
$$

Then, there exists $n \in \omega$ such that

$$
\mathrm{I} \Sigma_{1} \vdash \forall Z \subseteq \text { fin }(0, \infty)_{\mathbb{N}}\left(Z \text { is } \omega^{n} \text {-large } \rightarrow \exists F \subseteq Z \psi(F)\right) .
$$

Proof. By Lemma 2.3, any $\omega^{n+1}$-large set is $n$-dense $(0=0)$ (dense for the trivial statement). Thus, we have this corollary as a special case of Theorem 3.4 .

Note that this corollary quickly implies (a weaker version of) the Parsons theorem (see, e.g., [12]), namely, any $\Pi_{2}$-statement provably in $\mathrm{I} \Sigma_{1}$ is bounded by a primitive recursive function, as follows. If a $\Pi_{2}$-statement $\forall x \exists y \theta(x, y)$ is provable within $\mathrm{I} \Sigma_{1}^{0}$, then put $\psi(F): \equiv(\forall x<\min F)(\exists y<$ $\max F) \theta(x, y)$. Then, $\mathrm{I} \Sigma_{1}^{0}$ proves that any infinite set contains a finite set $F$ such that $\psi(F)$ holds. By this theorem, there exists $n \in \omega$ such that $\left(Z\right.$ is $\omega^{n}$-large $\left.\rightarrow \exists F \subseteq Z \psi(F)\right)$. One can easily find a primitive recursive function $h$ such that $[a, h(a)]_{\mathbb{N}}$ is $\omega^{n}$-large for any $a \in \omega$. Thus, we have $\mathrm{I} \Sigma_{1} \vdash \forall x \exists y<h(x) \theta(x, y)$. Note also that one can apply the generalized Parsons theorem for any $\tilde{\Pi}_{3}^{0}$-conservative extension of $\mathrm{I} \Sigma_{1}^{0}$, e.g., $\mathrm{WKL} L_{0}+\mathrm{B} \Sigma_{2}^{0}$.

We are now ready to prove the main conservation theorem of the section.

Proof of Theorem 3.1. Let $\Gamma$ be a Ramsey-like- $\Pi_{2}^{1}$-statement, and assume that for any $k \in \omega$,

$$
\mathrm{I} \Sigma_{1}^{0} \vdash \forall X \subseteq \mathbb{N}\left(X \text { is infinite } \rightarrow \exists F \subseteq \text { fin } X\left(F \text { is } \omega^{k} \text {-large }(\Gamma)\right)\right) .
$$

Then, by Corollary 3.5, for each $k \in \omega$ there exists $n_{k}$ such that

$$
\mathrm{I} \Sigma_{1}^{0} \vdash \forall Z \subseteq \text { fin }(0, \infty)_{\mathbb{N}}\left(Z \text { is } \omega^{n_{k}} \text {-large } \rightarrow Z \text { is } \omega^{k} \text {-large }(\Gamma)\right) \text {. }
$$

Now, put $h: \omega \rightarrow \omega$ as $h(0)=1$ and $h(m+1)=\max \left\{n_{h(m)}, h(m)+1\right\}$. We will check

$$
\mathrm{I} \Sigma_{1}^{0} \vdash \forall Z \subseteq_{\text {fin }}(0, \infty)_{\mathbb{N}}\left(Z \text { is } \omega^{h(m)} \text {-large } \rightarrow Z \text { is } m \text {-dense }(\Gamma)\right) .
$$

by induction. The case $m=0$ follows from the definition. The case $m=m^{\prime}+1, \omega^{h\left(m^{\prime}\right)}$-large sets are $m^{\prime}$-dense $(\Gamma)$ by the induction hypothesis. Then, the first condition of the $m^{\prime}+1$-density follows from $h\left(m^{\prime}+1\right) \geq n_{h\left(m^{\prime}\right)}$, and the second condition follows from $h\left(m^{\prime}+1\right) \geq h\left(m^{\prime}\right)+1$ and Lemma 2.3. Thus, by Theorem $2.4, \mathrm{I} \Sigma_{1}^{0}$ proves that any infinite set contains an $m$-dense $(\Gamma)$ set for any $m \in \omega$. Hence, by Theorem 3.3, $\mathrm{WKL}_{0}+\Gamma$ is a $\tilde{\Pi}_{3}^{0}$-conservative extension of $\mathrm{I} \Sigma_{1}^{0}$.

When two conservation results are obtained, one can often amalgamate those results. For example, if two $\Pi_{2}^{1}$-theories $T_{1}$ and $T_{2}$ are $\Pi_{1}^{1}$-conservative over a base $\Pi_{2}^{1}$-theory $T_{0}$, then $T_{1}+T_{2}$ is also $\Pi_{1}^{1}$-conservative over $T_{0}$ (see [64]). Similar amalgamation property holds for $\tilde{\Pi}_{3}^{0}$-conservation as follows. 
Theorem 3.6 (Amalgamation). Fix $n \geq 1$. Let $T$ be a theory extending $\mathrm{I} \Sigma_{1}^{0}$ which consists of sentences of the form $\forall X \exists Y \theta(X, Y)$ where $\theta$ is $\Pi_{n+2}^{0}$, and let $\Gamma_{1}$ and $\Gamma_{2}$ be sentences of the same form as $T$. If $T+\Gamma_{i}$ is a $\tilde{\Pi}_{n+2}^{0}$-conservative extension of $T$ for $i=1,2$, then, $T+\Gamma_{1}+\Gamma_{2}$ is a $\tilde{\Pi}_{n+2}^{0}$-conservative extension of $T$.

Proof. The proof is essentially the same as the case for the amalgamation of two $\Pi_{2}^{1}$-theories which are $\Pi_{1}^{1}$-conservative over a base theory in [64]. Here, we consider the $\mathcal{L}_{2}$-structure $(M, S)$ as a two-sorted structure, namely, $M$ and $S$ are disjoint sets and $\epsilon$ is a relation on $M \times S$. In this understanding, $\tilde{\Pi}_{n+2}^{0}$-conservation implies that any model of $T$ has a $\Sigma_{n+2}^{0}$-elementary extension which is a model of $T+\Gamma_{i}$ for $i=1,2$. (This is because if $(M, S) \models T$, then $\operatorname{Th}_{\mathcal{L}_{2} \cup M \cup S}(M, S) \cap$ $\Sigma_{n+2}^{0}+T+\Gamma_{i}$ is consistent.) Now, assume $T \forall \forall X \psi(X)$ where $\psi$ is $\Pi_{n+2}^{0}$, and take a model $\left(M_{0}, S_{0}\right) \models T+\exists X \neg \psi(X)$. Then, one can construct a $\Sigma_{n+2}^{0}$-elementary chain of models $\left(M_{0}, S_{0}\right) \subseteq$ $\left(M_{1}, S_{1}\right) \subseteq \ldots$ such that $\left(M_{2 j+i}, S_{2 j+i}\right) \models T+\Gamma_{i}$ for $i=1,2$ and $j \in \omega$. By the usual elementary chain argument, $(\bar{M}, \bar{S})=\left(\bigcup_{k \in \omega} M_{k}, \bigcup_{k \in \omega} S_{k}\right)$ is a $\Sigma_{n+2}^{0}$-elementary extension of $\left(M_{0}, S_{0}\right)$, and therefore $(\bar{M}, \bar{S}) \models T+\Gamma_{1}+\Gamma_{2}+\exists X \neg \psi(X)$. Hence $T+\Gamma_{1}+\Gamma_{2} \forall \forall \forall X \psi(X)$.

Note that $\mathrm{I}_{1}^{0}, \mathrm{WKL}_{0}$ and any Ramsey-like statement is of the form $\forall X \exists Y \theta(X, Y)$ where $\theta$ is $\Pi_{3}^{0}$. Therefore, one can always use the amalgamation theorem for $\tilde{\Pi}_{3}^{0}$-conservation. In particular, to prove the $\tilde{\Pi}_{3}^{0}$-conservation theorem for $\mathrm{RT}_{2}^{2}$, we only need to prove $\tilde{\Pi}_{3}^{0}$-conservation theorems for $\mathrm{ADS}$ and $\mathrm{EM}$.

\section{Conservation theorem for ADS}

In this section, we will show that $\mathrm{WKL}_{0}+\mathrm{ADS}$ is a $\tilde{\Pi}_{3}^{0}$-conservative extension of $\mathrm{I} \Sigma_{1}^{0}$. Actually, this is just a weakening of the following theorem by Chong, Slaman and Yang, where CAC is the chain antichain principle, since ADS is a consequence of CAC over RCA $\mathrm{A}_{0}$ [32].

Theorem 4.1 (Chong, Slaman, Yang [16]). $W L_{0}+\mathrm{CAC}$ is a $\Pi_{1}^{1}$-conservative extension of $\mathrm{B} \Sigma_{2}^{0}$.

Here, we will give an alternative proof by calculating the size of $\omega^{k}$-large(psRT $\left.{ }_{2}^{2}\right)$ sets. To simplify the proof below, we will use a slightly modified $\alpha$-largeness notion.

Definition $4.1\left(\mathrm{I} \Sigma_{1}^{0}\right)$. Any set is said to be 0 -large*. Given some $\alpha<\omega^{\omega}, X \subseteq_{\text {fin }} \mathbb{N}$ is said to be $\alpha$-large* if

- $X \backslash\{\min X\}$ is $\beta$-large* if $\alpha=\beta+1$,

- $X$ is $\left(\beta+\omega^{n-1} \cdot \min X\right)$-large* if $\alpha=\beta+\omega^{n}$.

Trivially, if $X \subseteq$ fin $\mathbb{N}$ is $\alpha$-large, then $X$ is $\alpha$-large*

Lemma 4.2. For any $k \in \omega$, the following is provable within $\mathrm{I} \Sigma_{1}^{0}$. For any $\alpha<\omega^{k}$ and for any $X \subseteq_{\text {fin }} \mathbb{N}, X$ is $\alpha$-large if $X$ is $\alpha+1$-large*.

Proof. By $\Pi_{1}^{0}$-transfinite induction up to $\omega^{k}$, which is available within $\mathrm{RCA}$.

Lemma 4.3. The following is provable within $\mathrm{I} \Sigma_{1}^{0}$. For any $k, n \in \mathbb{N}$, if $X$ is a disjoint union of $X_{0}, \ldots, X_{k-1}$ such that $X_{i}<X_{i+1}$ for any $i<k-1$ and each $X_{i}$ is $\omega^{n}$-large*, then $X$ is $\omega^{n} \cdot k$-large $e^{*}$ Thus, if $k \leq \min X_{0}, X$ is $\omega^{n+1}$-large $e^{*}$.

Proof. Similar to Lemma 2.3. 
Lemma 4.4. For any $k \in \omega$, the following is provable within $\mathrm{I} \Sigma_{1}^{0}$. If a finite set $X \subseteq \mathbb{N}$ is $\omega^{2 k+6}$-large and $\min X>3$, then $X$ is $\omega^{k}$-large $\left(\mathrm{psRT}_{2}^{2}\right)$.

Proof. Given a coloring $f:[X]^{2} \rightarrow 2$, define the coloring $\bar{f}:[X]^{2} \rightarrow 2 k+2$ as $\bar{f}(x, y)=2 j+i$ if $f(x, y)=i$ and $j=\min \left\{j^{\prime}<k \mid \neg\left(\exists H \subseteq[x, y)_{\mathbb{N}} \cap X x \in H, H\right.\right.$ is $\omega^{j^{\prime}+1}$-large ${ }^{*}$ and $H \cup\{y\}$ is pseudo-homogeneous for $f$ with the color $i)\} \cup\{k\}$.

By Theorem 2.6, take $Y \subseteq X$ such that $Y$ is $\omega$-large and $\bar{f}$-homogeneous. Let $Y=\left\{y_{0}<y_{1}<\right.$ $\left.\cdots<y_{l}\right\}$, and $\bar{f}\left([Y]^{2}\right)=2 j+i$. Then, $l \geq y_{0}$. By the definition of $\bar{f}$, for $s=0, \ldots, l-1$, one can take $H_{s} \subseteq\left[y_{s}, y_{s+1}\right)_{\mathbb{N}}$ such that $y_{s} \in H_{s}, H_{s}$ is $\omega^{j}$-large* and $\left[H_{s} \cup\left\{y_{s+1}\right\}\right]^{2}$ is pseudo-homogeneous for $f$ with the color $i$. By Lemma 4.3, $H=\bigcup_{s=0}^{l-1} H_{s}$ is $\omega^{j+1}$-large*, and $\left[H \cup\left\{y_{k}\right\}\right]^{2}$ is pseudohomogeneous for $f$ with the color $i$. This $H$ assures that $\bar{f}\left(y_{0}, y_{k}\right) \neq 2 j+i$ or $j=k$. Thus, we have $j=k$. Hence $H$ is pseudo-homogeneous for $f$ and $\omega^{k+1}$-large*, thus it is $\omega^{k}$-large.

Corollary 4.5. $\mathrm{WKL}_{0}+\mathrm{psRT}_{2}^{2}$, or equivalently, $\mathrm{WKL}_{0}+\mathrm{ADS}$ is a $\tilde{\Pi}_{3}^{0}$-conservative extension of $\mathrm{I} \Sigma_{1}^{0}$.

Proof. By Theorems 2.4, 3.1 and Lemma 4.4.

Note that we could have proven Corollary 4.5 by working with ADS directly. However, the unnatural formulation of ADS as a Ramsey-like- $\Pi_{2}^{1}$-statement introduces additional technicalities in the proof. Indeed, the standard formulation of ADS involves linear orders, whereas a Ramseylike statement is about arbitrary coloring functions. In this framework, a solution to ADS is either an infinite homogeneous set, or a set whose minimal element witnesses the non-transitivity of the coloring.

\section{Grouping principle}

In this section, we introduce the grouping principle, which is a consequence of Ramsey's theorem. The grouping principle will be used in the conservation proof of the Erdős-Moser theorem, although it is currently unknown how the two statements relate in reverse mathematics. The grouping principle seems interesting to study in its own right, and we conduct a study of its relations with other Ramsey-type principles already studied in reverse mathematics.

Definition 5.1 (RCA $A_{0}$, grouping principle). Given a largeness notion $\mathrm{L}$ and a coloring $f:[\mathbb{N}]^{n} \rightarrow k$, an L-grouping for $f$ is an infinite family of L-large finite sets $\left\{F_{0}<F_{1}<\ldots\right\} \subseteq \mathrm{L}$ such that

$$
\forall i_{1}<\cdots<i_{n} \exists c<k \forall x_{1} \in F_{i_{1}}, \ldots, \forall x_{n} \in F_{i_{n}} f\left(x_{1}, \ldots, x_{n}\right)=c
$$

Now $\operatorname{GP}_{k}^{n}(\mathrm{~L})$ (grouping principle for $\mathrm{L}$ ) asserts that for any coloring $f:[\mathbb{N}]^{n} \rightarrow k$, there exists an infinite $\mathrm{L}$-grouping for $f$. We write $\mathrm{GP}_{k}^{n}$ for the statement saying that for any largeness notion $\mathrm{L}$, $\mathrm{GP}_{k}^{n}(\mathrm{~L})$ holds, $\mathrm{GP}^{n}$ for $\forall k \mathrm{GP}_{k}^{n}$, and GP for $\forall n \mathrm{GP}^{n}$.

Note that being a largeness notion is a $\Pi_{1}^{1}$-statement. Therefore, an instance of $\mathrm{GP}_{2}^{2}$ is a pair $\langle\mathrm{L}, f\rangle$ where $\mathrm{L}$ is a collection of finite sets, and $f:[\mathbb{N}]^{2} \rightarrow 2$ is a coloring. A solution to an instance $\langle\mathrm{L}, f\rangle$ is either an $\mathrm{L}$-grouping for $f$, or an infinite set witnessing that $\mathrm{L}$ is not a largeness notion, that is, an infinite set with no finite subset in L.

In order to simplify the analysis of Ramsey's theorem for pairs, Cholak, Jockusch and Slaman [13] split the proof of $\mathrm{RT}_{2}^{2}$ into cohesiveness and a stable restriction of $\mathrm{RT}_{2}^{2}$. A coloring $f:[\mathbb{N}]^{2} \rightarrow k$ is stable if for every $x, \lim _{y} f(x, y)$ exists. $\mathrm{SRT}_{k}^{2}$ is the restriction of $\mathrm{RT}_{2}^{2}$ to stable colorings. 
Definition 5.2 (Cohesiveness). An infinite set $C$ is $\vec{R}$-cohesive for a sequence of sets $R_{0}, R_{1}, \ldots$ if for each $i \in \mathbb{N}, C \subseteq^{*} R_{i}$ or $C \subseteq^{*} \overline{R_{i}}$. COH is the statement "Every uniform sequence of sets $\vec{R}$ has an $\vec{R}$-cohesive set."

Cohesiveness is a statement from standard computability theory. Cholak, Jockusch and Slaman [13] claimed with an erroneous proof that it is a strict consequence of $\mathrm{RT}_{2}^{2}$ over $\mathrm{RCA}_{0}$. Mileti [45] fixed the proof. Hirschfeldt and Shore [32] proved that $\mathrm{COH}$ is a consequence of ADS. Since then, many statements in reverse mathematics have been split into their cohesive and their stable part [32]. Accordingly, we will consider the stable version $\mathrm{SGP}_{2}^{2}$ which stands for $\mathrm{GP}_{2}^{2}$ for stable colorings. One can prove that $\mathrm{RCA}_{0} \vdash \mathrm{COH}+\mathrm{SGP}_{2}^{2} \rightarrow \mathrm{GP}_{2}^{2}$ by the same argument as $\mathrm{RCA}_{0} \vdash \mathrm{COH}+\mathrm{SRT}_{2}^{2} \rightarrow \mathrm{RT}_{2}^{2}$ [13]. Stable Ramsey's theorem for pairs admits a nice computability-theoretic characterization in terms of infinite subsets of a $\Delta_{2}^{0}$ set. We can give a similar characterization for the stable grouping principle for pairs.

Definition 5.3 $\left(\mathrm{RCA}_{0}\right)$. Given a largeness notion $\mathrm{L}$, an $\mathrm{L}$-grouping for a set $A$ is an infinite family of L-large finite sets $\left\{F_{0}<F_{1}<\ldots\right\} \subseteq \mathrm{L}$ such that $(\forall i)\left[F_{i} \subseteq A \vee F_{i} \subseteq \bar{A}\right]$

The argument can be carried-out within $\mathrm{B} \Sigma_{2}^{0}$. Actually, Kreuzer proved that $\mathrm{SGP}_{2}^{2}$ implies $\mathrm{B} \Sigma_{2}^{0}$ over RCA (see Theorem 9.1).

Lemma 5.1. $\mathrm{RCA}_{0}+\mathrm{B} \Sigma_{2}^{0} \vdash \mathrm{SGP}_{2}^{2}(\mathrm{~L}) \leftrightarrow$ "Every $\Delta_{2}^{0}$ set has an infinite $\mathrm{L}$-grouping".

We will now show the existence of an $\omega$-model of $\mathrm{SGP}_{2}^{2}$ containing only low sets. Recall that an instance is a pair $\langle\mathrm{L}, f\rangle$, and a solution is either a witness that $\mathrm{L}$ is not a largeness notion, or an $\mathrm{L}$-grouping for $f$. We need therefore to show that given any computable collection of finite sets $\mathrm{L}$ and any $\Delta_{2}^{0}$ set $A$, there is either an infinite low set $Y$ witnessing that $\mathrm{L}$ is not a largeness notion, or a low L-grouping for $A$. In what follows, we denote by LOW the collection of all low sets.

Theorem 5.2. For every computable set $\mathrm{L}$ which is a largeness notion on $(\omega, \mathrm{LOW})$, every $\Delta_{2}^{0}$ set has an infinite low L-grouping.

Proof. Fix a $\Delta_{2}^{0}$ set $A$. We will construct an infinite low L-grouping for $A$ by an effective forcing notion whose conditions are tuples $c=\left(F_{0}, \ldots, F_{k}, X_{0}, \ldots, X_{m}\right)$ such that

(i) $F_{i} \in \mathrm{L}$ and $F_{i} \subseteq_{\text {fin }} A$ or $F_{i} \subseteq_{\text {fin }} \bar{A}$ for each $i \leq k$,

(ii) $F_{i}<F_{i+1}$ for each $i<k$,

(iii) $X_{0} \sqcup \cdots \sqcup X_{m}$ is a low partition of $\omega$.

A condition $d=\left(F_{0}, \ldots, F_{\ell}, Y_{0}, \ldots, Y_{n}\right)$ extends a condition $c=\left(F_{0}, \ldots, F_{k}, X_{0}, \ldots, X_{m}\right)$ (written $d \leq c)$ if $\ell \geq k$, for every $i \in(k, \ell]_{\mathbb{N}}, F_{i} \subseteq X_{j}$ for some $j \leq m$ and $Y_{0}, \ldots, Y_{n}$ refines $X_{0}, \ldots, X_{m}$, that is, for each $i \leq n$, there is some $j \leq m$ such that $Y_{i} \subseteq X_{j}$. An index of the condition $c$ is a tuple $\left(F_{0}, \ldots, F_{k}, e\right)$ where $\Phi_{e}^{\emptyset^{\prime}}$ decides the jump of the partition $X_{0}, \ldots, X_{m}$. We first claim that the finite sequence of sets can be extended.

Claim. For every condition $c=\left(F_{0}, \ldots, F_{k}, X_{0}, \ldots, X_{m}\right)$, there is an extension $d=\left(F_{0}, \ldots, F_{\ell}\right.$, $\left.X_{0}, \ldots, X_{m}\right)$ of $c$ such that $\ell>k$. Moreover, an index of $d$ can be found $\emptyset^{\prime}$-uniformly in an index of $c$. 
Proof of the claim. We first show that there is a set $F>F_{k}$ such that $F \in \mathrm{L}$ and $F \subseteq X_{i} \cap A$ or $F \subseteq X_{i} \cap \bar{A}$ for some $i \leq n$. Let $i \leq n$ be such that $X_{i}$ is infinite. We claim that there is some finite set $F \in \mathrm{L}$ such that $F \subseteq X_{i} \cap A \backslash\left[0, \max \left(F_{k}\right)\right]_{\mathbb{N}}$ or $F \subseteq X_{i} \cap \bar{A} \backslash\left[0, \max \left(F_{k}\right)\right]_{\mathbb{N}}$. Suppose for the sake of contradiction that there is no such set. Then the $\Pi_{1}^{0, X_{i}}$ class of all sets $Z$ such that for every $F \in \mathrm{L}, F \nsubseteq X_{i} \cap Z \backslash\left[0, \max \left(F_{k}\right)\right]_{\mathbb{N}}$ and $F \nsubseteq X_{i} \cap \bar{Z} \backslash\left[0, \max \left(F_{k}\right)\right]_{\mathbb{N}}$ is non-empty. By the low basis theorem, there is a $Z$ such that $Z \oplus X_{i}$ is low over $X_{i}$, hence low. The set $Z$ or its complement contradicts the fact that $\mathrm{L}$ is a largeness notion on $(\omega, \mathrm{LOW})$.

Knowing that such a set $F$ exists, we can find it $\emptyset^{\prime}$-uniformly in $c$ and a $\Delta_{2}^{0}$ index of the set $A$. The condition $\left(F_{0}, \ldots, F_{k}, F, X_{0}, \ldots, X_{m}\right)$ is a valid extension of $c$. Note that such a set $F$ does not need to be part of an infinite $X_{i} \cap A$ or $X_{i} \cap \bar{A}$. The choice of an infinite part has simply been used to claim the existence of any such set.

We say that an L-grouping for $A\left\langle E_{0}<E_{1}<\ldots\right\rangle$ satisfies a condition $c=\left(F_{0}, \ldots, F_{k}, X_{0}, \ldots, X_{m}\right)$ if $E_{0}=F_{0}, \ldots, E_{k}=F_{k}$ and for every $i>k$, there is some $j \leq m$ such that $E_{i} \subseteq X_{j}$. A condition $c$ forces formula $\varphi(G)$ if $\varphi(\vec{E})$ holds for every L-grouping $\vec{E}$ satisfying $c$.

Claim. For every condition c and every index $e \in \omega$, there is an extension $d$ forcing either $\Phi_{e}^{G}(e) \downarrow$ or $\Phi_{e}^{G}(e) \uparrow$. Moreover, an index of $d$ can be found $\emptyset^{\prime}$-uniformly in an index of $c$ and $e$.

Proof of the claim. Fix a condition $c=\left(F_{0}, \ldots, F_{k}, X_{0}, \ldots, X_{m}\right)$. We have two cases.

In the first case, for every 2-partition $Z_{0} \cup Z_{1}=\omega$, there is a sequence of finite sets $F_{k+1}, \ldots, F_{\ell}$ such that $F_{k}<F_{k+1}<\cdots<F_{\ell}, \Phi_{e}^{F_{0}, \ldots, F_{\ell}}(e) \downarrow$, and for every $i \in(k, \ell], F_{i} \in \mathrm{L}$ and there is some $j \leq m F_{i} \subseteq Z_{0} \cap X_{j}$ or $F_{i} \subseteq Z_{1} \cap X_{j}$. In particular, taking $Z_{0}=A$ and $Z_{1}=\bar{A}$, there is a sequence of finite sets $F_{k+1}, \ldots, F_{\ell}$ such that $d=\left(F_{0}, \ldots, F_{\ell}, X_{0}, \ldots, X_{m}\right)$ is a valid extension of $c$ and $\Phi_{e}^{F_{0}, \ldots, F_{\ell}}(e) \downarrow$. Such an extension can be found $\emptyset^{\prime}$-uniformly in an index of $c, e$ and a $\Delta_{2}^{0}$ index of $A$.

In the second case, the $\Pi_{1}^{0, \vec{X}}$ class of all the 2-partitions $Z_{0} \cup Z_{1}=\omega$ such that $\Phi_{e}^{F_{0}, \ldots, F_{e}}(e) \uparrow$ for every sequence of finite sets $F_{k+1}, \ldots, F_{\ell}$ such that $F_{k}<F_{k+1}<\cdots<F_{\ell}$, and for every $i \in(k, \ell]_{\mathbb{N}}$, $F_{i} \in \mathrm{L}$ and there is some $j \leq m F_{i} \subseteq Z_{0} \cap X_{j}$ or $F_{i} \subseteq Z_{1} \cap X_{j}$ is non-empty. By the low basis theorem [35] relativized to $\vec{X}$, there is a such a 2-partition $Z_{0} \cup Z_{1}=\omega$ such that $Z_{0} \oplus Z_{1} \oplus \vec{X}$ is low. Moreover, a lowness index for $Z_{0} \oplus Z_{1} \oplus \vec{X}$ can be found uniformly in a lowness index for $\vec{X}$. The condition $d=\left(F_{0}, \ldots, F_{k}, X_{0} \cap Z_{0}, X_{0} \cap Z_{1}, \ldots, X_{m} \cap Z_{0}, X_{m} \cap Z_{1}\right)$ is an extension of $c$ forcing $\Phi_{e}^{G}(e) \uparrow$.

Moreover, we can $\vec{X}^{\prime}$-decide (hence $\emptyset^{\prime}$-decide) whether the $\Pi_{1}^{0, \vec{X}}$ class is empty, thus we can find the extension $d \emptyset^{\prime}$-effectively in an index of $c$ and $e$.

Thanks to the claims, define an infinite, uniformly $\emptyset^{\prime}$-computable decreasing sequence of conditions $c_{0}=(\varepsilon, \omega) \geq c_{1} \geq c_{2} \geq \ldots$, where $c_{s}=\left(F_{0}, \ldots, F_{k_{s}}, X_{0}^{s}, \ldots, X_{m_{s}}^{s}\right)$ such that for each $s \in \omega$

(a) $k_{s} \geq s$

(b) $c_{s+1}$ forces $\Phi_{s}^{G}(s) \uparrow$ or $\Phi_{s}^{G}(s) \downarrow$

This sequence yields a L-grouping for $A\left\langle F_{0}, F_{1}, \ldots\right\rangle$ which is infinite by (a) and whose jump is $\Delta_{2}^{0}$ by (b). This finishes the proof of Theorem 5.2.

Corollary 5.3. $\mathrm{SGP}_{2}^{2}+\mathrm{SADS}+\mathrm{WKL}_{0}$ has an $\omega$-model with only low sets. 
Proof. By Lemma 5.1 and and the low basis theorem [35] in a relativized form. As explained, for every collection $L$ and every stable coloring $f:[\omega]^{2} \rightarrow 2$, one need either to add a low set witnessing that $\mathrm{L}$ is not a notion of largeness, or to add an infinite low $\mathrm{L}$-grouping for $f$.

Corollary 5.4. $\mathrm{RCA}_{0}+\mathrm{SGP}_{2}^{2}+\mathrm{SADS}+\mathrm{WKL}_{0}$ implies neither $\mathrm{SRT}_{2}^{2}$, nor $\mathrm{SEM}$.

Proof. Downey, Hirschfeldt, Lempp and Solomon [20] built a computable instance of $\mathrm{SRT}_{2}^{2}$ with no low solution. Corollary 5.3 enables us to conclude that $\mathrm{RCA}_{0}+\mathrm{SGP}_{2}^{2}+\mathrm{SADS}+\mathrm{WKL}_{0}$ does not imply $\mathrm{SRT}_{2}^{2}$. Since RCA $\mathrm{A}_{0}+\mathrm{SADS}+\mathrm{SEM}$ implies $\mathrm{SRT}_{2}^{2}$ (see [43]) then $\mathrm{RCA}_{0}+\mathrm{SGP}_{2}^{2}+\mathrm{SADS}+\mathrm{WKL}_{0}$ does not imply SEM.

Among the computability-theoretic properties used to separate Ramsey-type theorems in reverse mathematics, the framework of preservation of hyperimmunity has been especially fruitful.

Definition 5.4 (Hyperimmunity). The principal function of a set $B=\left\{x_{0}<x_{1}<\ldots\right\}$ is the function $p_{B}$ defined by $p_{B}(i)=x_{i}$ for each $i \in \mathbb{N}$. A set $X$ is hyperimmune if its principal function is not dominated by any computable function.

Wang [62] recently used the notion of preservation of the arithmetic hierarchy to separate various theorems in reverse mathematics. The first author showed [50] that a former separation of the Erdős-Moser theorem from stable Ramsey's theorem for pairs due to Lerman, Solomon and Towsner [43] could be reformulated in a similar framework, yielding the notion of preservation of hyperimmunity.

Definition 5.5 (Preservation of hyperimmunity). A $\Pi_{2}^{1}$-statement $\mathrm{P}$ admits preservation of hyperimmunity if for each set $Z$, each $Z$-hyperimmune sets $A_{0}, A_{1}, \ldots$, and each P-instance $X \leq_{T} Z$, there is a solution $Y$ to $X$ such that the $A$ 's are $Y \oplus Z$-hyperimmune.

In particular, if a $\Pi_{2}^{1}$-statement $P$ admits preservation of hyperimmunity but another statement $Q$ does not, then $P$ does not imply $Q$ over $R C A_{0}$. We now show that the grouping principle enjoys preservation of hyperimmunity and deduce several separations from it.

Theorem 5.5. $\mathrm{SGP}_{2}^{2}$ admits preservation of hyperimmunity.

Proof. Let $C$ be a set and $B_{0}, B_{1}, \ldots$ be a sequence of $C$-hyperimmune sets. Let $S$ be the collection of all sets $X$ such that the $B$ 's are $X \oplus C$-hyperimmune. By Lemma 5.1, it suffices to show that for every $\Delta_{2}^{0, C}$ set $A$ and every $C$-computable largeness notion $\mathrm{L}$ on $(\omega, S)$ there is an infinite L-grouping $\vec{F}=\left\langle F_{0}<F_{1}<\ldots\right\rangle$ for $A$ such that the $B$ 's are $\vec{F} \oplus C$-hyperimmune. Therefore, every instance $\langle\mathrm{L}, A\rangle$ will have a solution $Y \in S$, which will be either a witness that $\mathrm{L}$ is not a largeness notion, or an L-grouping for $A$.

Fix $A$ and L. We will construct an infinite L-grouping for $A$ by a forcing argument whose conditions are tuples $\left(F_{0}, \ldots, F_{k}, X\right)$ where

(i) $F_{i} \in \mathrm{L}$ and $F_{i} \subseteq_{\text {fin }} A$ or $F_{i} \subseteq_{\text {fin }} \bar{A}$ for each $i \leq k$.

(ii) $F_{i}<F_{i+1}$ for each $i<k$

(iii) $X$ is an infinite set such that the $B$ 's are $X \oplus C$-hyperimmune.

A condition $d=\left(F_{0}, \ldots, F_{\ell}, Y\right)$ extends a condition $c=\left(F_{0}, \ldots, F_{k}, X\right)$ (written $\left.d \leq c\right)$ if $\ell \geq k$ and for every $i \in(k, \ell]_{\mathbb{N}}, F_{i} \subseteq X$. The proof of the following claim is exactly the same as in Theorem 5.2, using the hyperimmune-free basis theorem instead of the low basis theorem. 
Claim. For every condition $c=\left(F_{0}, \ldots, F_{k}, X\right)$, there is an extension $d=\left(F_{0}, \ldots, F_{\ell}, Y\right)$ of $c$ such that $\ell>k$.

The following claim shows that every sufficiently generic filter yields a sequence $\vec{F}$ such that the $B$ 's are $\vec{F} \oplus C$-hyperimmune. The notion of satisfaction and of forcing a formula $\varphi(G)$ are defined as in Theorem 5.2.

Claim. For every condition $c$ and every pair of indices $e, i \in \omega$, there is an extension $d$ forcing $\Phi_{e}^{G \oplus C}$ not to dominate $p_{B_{i}}$.

Proof of the claim. Fix a condition $c=\left(F_{0}, \ldots, F_{k}, X\right)$. Let $f$ be the function which on input $x$, searches for a finite set of integers $U$ such that for every 2-partition $Z_{0} \cup Z_{1}=X$, there is some finite sequence of sets $F_{k+1}, \ldots, F_{\ell}$ such that $F_{k}<\cdots<F_{\ell}, \Phi_{e}^{\left(F_{0}, \ldots, F_{\ell}\right) \oplus C}(x) \downarrow \in U$ and for every $i \in(k, \ell]_{\mathbb{N}}, F_{i} \in \mathrm{L} \cap Z_{0}$ or $F_{i} \in \mathrm{L} \cap Z_{1}$. If such a set $U$ is found, $f(x)=1+\max (U)$, otherwise $f(x) \uparrow$. The function $f$ is partial $X \oplus C$-computable. We have two cases.

- Case 1: $f$ is total. By $X \oplus C$-hyperimmunity of $B_{i}$, there is some $x$ such that $f(x) \leq p_{B_{i}}(x)$. Let $U$ be the finite set witnessing $f(x) \downarrow$. By taking $Z_{0}=X \cap A$ and $Z_{1}=X \cap \bar{A}$, there is a finite sequence of sets $F_{k+1}, \ldots, F_{\ell}$ such that $\Phi_{e}^{\left(F_{0}, \ldots, F_{\ell}\right) \oplus C}(x) \downarrow \in U$ and $d=\left(F_{0}, \ldots, F_{\ell}, X\right)$ is a valid extension of $c$. The condition $d$ forces $\Phi_{e}^{G \oplus C}(x)<f(x)$.

- Case 2: there is some $x$ such that $f(x) \uparrow$. By compactness, the $\Pi_{1}^{0, X \oplus C}$ class $\mathcal{C}$ of sets $Z_{0} \oplus Z_{1}$ such that $Z_{0} \cup Z_{1}=X$ and $\Phi_{e}^{\left(F_{0}, \ldots, F_{\ell}\right) \oplus C}(x) \uparrow$ for every finite sequence of sets $F_{k+1}, \ldots, F_{\ell}$ such that $F_{k}<\cdots<F_{\ell}$ and for every $i \in(k, \ell]_{\mathbb{N}}, F_{i} \in \mathrm{L} \cap Z_{0}$ or $F_{i} \in \mathrm{L} \cap Z_{1}$ is not empty. By the hyperimmune-free basis theorem [35], there exists some partition $Z_{0} \oplus Z_{1} \in \mathcal{C}$ such that the $B$ 's are $Z_{0} \oplus Z_{1} \oplus C$-hyperimmune. The set $Z_{j}$ is infinite for some $j<2$ and the condition $d=\left(F_{0}, \ldots, F_{k}, Z_{j}\right)$ is an extension of $c$ forcing $\Phi_{e}^{G \oplus C}(x) \uparrow$.

Let $\mathcal{F}$ be a sufficiently generic filter for this notion of forcing. The filter $\mathcal{F}$ yields a sequence $\vec{F}=$ $\left\langle F_{0}, F_{1}, \ldots\right\rangle$ which is infinite by the first claim, and such that the $B$ 's are $\vec{F} \oplus C$-hyperimmune by the second claim. This finishes the proof of Theorem 5.5.

Corollary 5.6. $\mathrm{RCA}_{0}+\mathrm{GP}_{2}^{2}+\mathrm{COH}+\mathrm{EM}+\mathrm{WKL}_{0}$ does not imply $\mathrm{ADS}$.

Proof. By the hyperimmune-free basis theorem [35], WKL $\mathrm{W}_{0}$ admits preservation of hyperimmunity. The first author proved in [50] that $\mathrm{COH}$ and $\mathrm{EM}$ admit preservation of hyperimmunity, but that $\mathrm{ADS}$ does not. Last, $\mathrm{GP}_{2}^{2}$ admits preservation of hyperimmunity since $\mathrm{COH}+\mathrm{SGP}_{2}^{2}$ implies $\mathrm{GP}_{2}^{2}$ over RCA .

Definition 5.6 (Diagonally non-computable function). A function $f$ is diagonally non-computable (d.n.c.) relative to $X$ if for every $e, f(e) \neq \Phi_{e}^{X}(e) .2$-DNC is the statement "For every set $X$, there is a function d.n.c. relative to the jump of $X$ ".

Beware, the notation 2-DNC may cause some confusion with $\mathrm{DNC}_{2}$, the restriction to $\{0,1\}$ valued d.n.c. functions which is equivalent to $W K L_{0}$ over $R C A_{0}$. The following proof is an adaptation of the proof that the Erdős-Moser implies 2-DNC over RCA [51].

Theorem 5.7. $\mathrm{RCA}_{0} \vdash \mathrm{GP}_{2}^{2}\left(\mathrm{~L}_{\omega}\right) \rightarrow 2$-DNC 
Proof. Fix a set $X$. Let $g(.,$.$) be a total X$-computable function such that $\Phi_{e}^{X^{\prime}}(e)=\lim _{s} g(e, s)$ if the limit exists, and $\Phi_{e}^{X^{\prime}}(e) \uparrow$ if the limit does not exist. Also fix for each $e \in \mathbb{N}$ an enumeration $D_{e, 0}, D_{e, 1}, \ldots$ of all finite sets of cardinal $3^{e+1}$. We define the function $f:[\mathbb{N}]^{2} \rightarrow 2$ by primitive recursion. Let $f_{0}$ be the function nowhere defined. At stage $s+1$, do the following. Start with $f_{s+1}=f_{s}$. Then, for each $e<s$, take the first pair $\{x, y\} \in\left[\left(D_{e, g(e, s)} \cap[0, s)_{\mathbb{N}}\right) \backslash \bigcup_{k<e} D_{k, g(k, s)}\right]^{2}$ if it exists, and set $f_{s+1}(x, s)=0$ and $f_{s+1}(y, s)=1$. Finally, set $f(z, s)=0$ for any $z<s$ such that $f_{s+1}(s, z)$ remains undefined. This finishes the construction of $f_{s+1}$. Note that $f_{s}$ is defined on $\left[[0, s]_{\mathbb{N}}\right]^{2}$. Thus, $f=\bigcup_{s} f_{s}$ must exist and is total on $[\mathbb{N}]^{2}$.

By $\mathrm{GP}_{2}^{2}\left(\mathrm{~L}_{\omega}\right)$, let $\vec{F}=\left\langle F_{0}, F_{1}, \ldots\right\rangle$ be an infinite $\mathrm{L}_{\omega}$-grouping for $f$. Let $h(e)$ be such that $D_{e, h(e)} \subseteq F$ for some $F \in \vec{F}$. Such an $F$ exists since $D_{e, 0}, D_{e, 1}, \ldots$ enumerates all finite sets of cardinality $3^{e+1}$, and $\vec{F}$ contains sets of arbitrary size. We claim that $h(e) \neq \Phi_{e}^{X^{\prime}}(e)$ for all $e$, which would prove 2-DNC. Suppose otherwise, i.e., suppose that $\Phi_{e}^{X^{\prime}}(e)=h(e)$ for some $e$. Let $F \in \vec{F}$ be such that $D_{e, h(e)} \subseteq F$. Then there is a stage $s_{0}$ such that $h(e)=g(e, s)$ for all $s \geq s_{0}$ or equivalently $D_{e, g(e, s)}=D_{e, h(e)} \subseteq F$ for all $s \geq s_{0}$. We claim that for any $s$ be bigger than both $\max (F)$ and $s_{0}$, there are some $x, y \in D_{e, h(e)} \subseteq F$ such that $f(x, s) \neq f(y, s)$, which contradicts the fact that $\vec{F}$ is an $\mathrm{L}_{\omega}$-grouping for $f$.

To see this, let $s$ be such a stage. At that stage $s$ of the construction of $f$, a pair $\{x, y\} \in$ $\left[\left(D_{e, g(e, s)} \cap[0, s)_{\mathbb{N}}\right) \backslash \bigcup_{k<e} D_{k, g(k, s)}\right]^{2}$ is selected by a cardinality argument since $\left|D_{e, s} \cap[0, s)_{\mathbb{N}}\right|=$ $\left|D_{e, s}\right|=3^{e+1}>\sum_{k<e} 3^{k+1}=\left|\bigcup_{k<e} D_{k, g(k, s)}\right|$. Since $D_{e, g(e, s)}=D_{e, h(e)} \subseteq F$, this pair is contained in $F$. At this stage, we set $f(x, s) \neq f(y, s)$, therefore, $\vec{F}$ is not an $\mathrm{L}_{\omega}$-grouping for $f$, contradiction.

In particular, $\mathrm{SRT}_{2}^{2}$ does not imply $\mathrm{GP}_{2}^{2}\left(\mathrm{~L}_{\omega}\right)$ over $\mathrm{RCA}_{0}$ since Chong, Slaman and Yang [15] built a (non-standard) model of $\mathrm{SRT}_{2}^{2}+\mathrm{B} \Sigma_{2}^{0}$ containing only low sets, whereas provably in $\mathrm{RCA}_{0}$, there is no $\Delta_{2}^{0}$ d.n.c. function relative to $\emptyset^{\prime}$.

Definition 5.7 (Rainbow Ramsey theorem). Fix $n, k \in \mathbb{N}$. A coloring function $f:[\mathbb{N}]^{n} \rightarrow \mathbb{N}$ is $k$-bounded if for every $y \in \mathbb{N}$, card $f^{-1}(y) \leq k$. A set $R$ is a rainbow for $f$ (or an $f$-rainbow) if $f$ is injective over $[R]^{n}$. $\operatorname{RRT}_{k}^{n}$ is the statement "Every $k$-bounded function $f:[\mathbb{N}]^{n} \rightarrow \mathbb{N}$ has an infinite $f$-rainbow".

Miller [46] proved that the statement 2-DNC is equivalent to the rainbow Ramsey theorem for pairs $\left(\mathrm{RRT}_{2}^{2}\right)$ over $\mathrm{RCA}_{0}$.

Corollary 5.8. $\mathrm{RCA}_{0} \vdash \mathrm{GP}_{2}^{2} \rightarrow \mathrm{RRT}_{2}^{2}$.

Seetapun and Slaman [55] defined a Cardinality scheme for a set of formulas $\Gamma$ as follows. For every $\varphi(x, y) \in \Gamma, \mathrm{C} \Gamma$ contains the universal closure of the formula "If $\varphi(x, y)$ defines an injective function, then its range is unbounded". Conidis and Slaman [19] proved that the rainbow Ramsey theorem for pairs implies the $\Sigma_{2}$ cardinality scheme $\left(C \Sigma_{2}\right)$.

Corollary 5.9. $\mathrm{RCA}_{0} \vdash \mathrm{GP}_{2}^{2} \rightarrow \mathrm{C}_{2}$.

In particular, this shows that $\mathrm{GP}_{2}^{2}$ is not $\Pi_{1}^{1}$-conservative over $\mathrm{RCA}_{0}+\mathrm{I} \Sigma_{1}^{0}$ since a Skolem hull argument shows that $\mathrm{C} \Sigma_{2}$ is not provable in $\mathrm{I} \Sigma_{1}$ (see Seetapun and Slaman [55]).

\section{Conservation theorem for $\mathrm{GP}_{2}^{2}$}

In this section, we will prove a conservation result for the grouping principle. To calculate the size of $\alpha$-large(EM) sets in Section 7, we will use a finite version of the grouping principle within 
$\mathrm{I} \Sigma_{1}^{0}$. Instead of proving the finite grouping principle within $\mathrm{I} \Sigma_{1}^{0}$ directly, we would like to show a conservation theorem for the infinite grouping principle over $\mathrm{I} \Sigma_{1}^{0}$.

In Section 5, we have seen that $\mathrm{SGP}_{2}^{2}$ has an $\omega$-model with only low sets. It is well-known that a low solution construction is often able to be converted into a forcing construction of a solution satisfying $\Sigma_{1}^{0}$-induction within a countable nonstandard model, which leads to a $\Pi_{1}^{1}$-conservation over $\mathrm{I} \Sigma_{1}^{0}$. Unfortunately, our construction of a low solution for $\mathrm{SGP}_{2}^{2}$ in Section 5 requires $\mathrm{B} \Sigma_{2}^{0}$ and thus it is not formalizable within $\mathrm{I} \Sigma_{1}^{0}$. To overcome this situation, we show a general conservation theorem characterized by using recursively saturated models.

Theorem 6.1. Fix $n \geq 1$. Let $\Gamma$ be a formula of the form $\forall X \exists Y \theta(X, Y)$ where $\theta$ is $\Pi_{n+1}^{0}$. Then, $\mathrm{RCA}_{0}+\mathrm{B} \Sigma_{n+1}^{0}+\Gamma$ is a $\tilde{\Pi}_{n+2}^{0}$-conservative extension of $\mathrm{I} \Sigma_{n}^{0}$ if the following condition holds:

(†) for any countable recursively saturated model $(M, S) \models \mathrm{B} \Sigma_{n+1}^{0}$ and for any $X \in S$, there exists $Y \subseteq M$ such that $(M, S \cup\{Y\}) \models \mathrm{I} \Sigma_{n}^{0}+\theta(X, Y)$.

To show this theorem, we use the following property of recursively saturated models and resplendent models, which are introduced by Barwise and Schlipf. See [4] for the historical information of recursively saturated models and resplendent models.

Theorem 6.2 (see Sections 1.8 and 1.9 of [38]). Let $\mathcal{L} \supseteq \mathcal{L}_{\mathrm{PA}}$ be a finite language, and let $\mathcal{M}$ be a countable $\mathcal{L}$-structure. Then the following are equivalent.

1. $\mathcal{M}$ is recursively saturated.

2. $\mathcal{M}$ is resplendent, i.e., for any recursive set of sentences $T$ of a finite language $\mathcal{L}^{\prime} \supseteq \mathcal{L}$ such that $\operatorname{Th}(\mathcal{M}) \cup T$ is consistent, there exists an expansion $\mathcal{M}^{\prime}$ of $\mathcal{M}$ such that $\mathcal{M}^{\prime} \models T$.

3. $\mathcal{M}$ is chronically resplendent, i.e., $\mathcal{M}$ is resplendent with the extra condition that the expansion $\mathcal{M}^{\prime}$ is resplendent as an $\mathcal{L}^{\prime}$-structure.

Proof of Theorem 6.1. Let $\Gamma \equiv \forall X \exists Y \theta(X, Y)$ where $\theta$ is $\Pi_{n+1}^{0}$ satisfy the condition ( $\left.\dagger\right)$, and let $\varphi_{0} \equiv \forall X \forall x \varphi(X, x)$ be a $\tilde{\Pi}_{n+2}^{0}$-sentence where $\varphi$ is $\Sigma_{n+1}^{0}$. We will show that $\Sigma_{n}^{0} \not \forall \varphi_{0}$ implies that $\mathrm{RCA}_{0}+\mathrm{B} \Sigma_{n+1}^{0}+\Gamma \nvdash \varphi_{0}$. Assume that $\mathrm{I} \Sigma_{n}^{0} \nvdash \varphi_{0}$, and take a countable recursively saturated model $(M, S) \models \mathrm{I} \Sigma_{n}^{0}$ such that $(M, S) \models \neg \varphi_{0}$. Then, there exists $a \in M$ and $A \in S$ such that $(M,\{A\}) \models \neg \varphi(A, a)$. We will construct an ( $\omega$-length) sequence of cuts $M=I_{0} \supseteq_{e} I_{1} \supseteq_{e} \ldots$ and a sequence of sets $A_{i} \subseteq I_{i}$ such that

- $\left(I_{i},\left\{A_{0}, \ldots, A_{i}\right\} \mid I_{i}\right) \models \mathrm{I} \Sigma_{n}^{0}$ and $\left(I_{i},\left\{A_{0}, \ldots, A_{i}\right\}\left\lceil I_{i}\right)\right.$ is recursively saturated,

- if $i<j$, then $\left(I_{j},\left\{A_{0}, \ldots, A_{i}\right\}\left\lceil I_{j}\right)\right.$ is a $\Sigma_{n}^{0}$-elementary substructure of $\left(I_{i},\left\{A_{0}, \ldots, A_{i}\right\} \mid I_{i}\right)$, and,

- $\left(I_{i+1},\left\{A_{0}, \ldots, A_{i}, Z_{i}, A_{i+1}\right\}\left\lceil I_{i+1}\right) \models \theta\left(Z_{i}, A_{i+1}\right)\right.$, where $Z_{i}$ is a $k$-th $\Delta_{1}^{0}$-definable set in $\left(I_{j},\left\{A_{0}, \ldots, A_{j}\right\} \mid I_{j}\right)$ if $i=(j, k)$.

Set $I_{0}=M$ and $A_{0}=A \oplus\{a\}$. Now, given $\left(I_{i},\left\{A_{0}, \ldots, A_{i}\right\} \mid I_{i}\right)$, we will first find a cut $I_{i+1} \subsetneq e$ $I_{i}$ such that $\left(I_{i+1},\left\{A_{0}, \ldots, A_{i}\right\}\left\lceil I_{i+1}\right)\right.$ is $\Sigma_{n}^{0}$-elementary substructure of $\left(I_{i},\left\{A_{0}, \ldots, A_{i}\right\}\left\lceil I_{i}\right)\right.$ and recursively saturated. Let $J_{0}$ be the set of all $\Sigma_{n}^{0}$-definable elements in $\left(I_{i},\left\{A_{0}, \ldots, A_{i}\right\}\left\lceil I_{i}\right)\right.$. Since $\left(I_{i},\left\{A_{0}, \ldots, A_{i}\right\}\left\lceil I_{i}\right)\right.$ is recursively saturated, $J_{0}$ is not cofinal in $I_{i}$, thus $J=\sup J_{0}$ forms a proper cut of $I_{i}$ and it is a $\Sigma_{n}^{0}$-elementary substructure. Therefore, a recursive $\mathcal{L}_{\mathrm{PA}} \cup\left\{A_{0}, \ldots, A_{i}, J\right\}$ theory $\mathrm{T}$ saying that $\left(J,\left\{A_{0}, \ldots, A_{i}\right\}\lceil J)\right.$ is a $\Sigma_{n}^{0}$-elementary proper cut of $\left(I_{i},\left\{A_{0}, \ldots, A_{i}\right\}\left\lceil I_{i}\right)\right.$ 
is consistent with $\operatorname{Th}\left(\left(I_{i},\left\{A_{0}, \ldots, A_{i}\right\} \backslash I_{i}\right)\right)$. (One can state $\Sigma_{n}^{0}$-elementarity by using the truth predicate.) Thus, by the chronic resplendency of Theorem 6.2, there exists $J^{\prime} \subseteq I_{i}$ such that $\left(I_{i},\left\{A_{0}, \ldots, A_{i}, J^{\prime}\right\} \mid I_{i}\right)$ satisfies $T$ and is recursively saturated. Let $I_{i+1}$ be such $J^{\prime}$, then $\left(I_{i+1},\left\{A_{0}\right.\right.$, $\left.\ldots, A_{i}\right\}\left\lceil I_{i+1}\right)$ is $\Sigma_{n}^{0}$-elementary substructure of $\left(I_{i},\left\{A_{0}, \ldots, A_{i}\right\}\left\lceil I_{i}\right)\right.$ and recursively saturated.

By Theorem 1.7, $\left(I_{i+1},\left\{A_{0}, \ldots, A_{i}\right\} \mid I_{i+1}\right) \models \mathrm{B} \Sigma_{n+1}^{0}$, and thus $\left(I_{i+1},\left\{A_{0}, \ldots, A_{i}, Z_{i}\right\} \mid I_{i+1}\right) \models$ $\mathrm{B} \Sigma_{n+1}^{0} \cdot\left(Z_{i} \cap I_{i+1}\right.$ is $\Delta_{1}^{0}$-definable in $\left(I_{i+1},\left\{A_{0}, \ldots, A_{i}\right\}\left\lceil I_{i+1}\right)\right.$ by $\Sigma_{n}^{0}$-elementarity. $)$ Thus, by the condition ( $\dagger)$, there exists $B \subseteq I_{i+1}$ such that $\left(I_{i+1},\left\{A_{0}, \ldots, A_{i}, Z_{i}, B\right\} \mid I_{i+1}\right) \models \theta\left(Z_{i}, B\right)$. By using chronic resplendency as above, one can re-choose $B \subseteq I_{i+1}$ so that $\left(I_{i+1},\left\{A_{0}, \ldots, A_{i}, Z_{i}, B\right\}\left\lceil I_{i+1}\right)\right.$ is recursively saturated. Then, put $A_{i+1}$ as such $B$.

Claim. $\bar{I}=\bigcap_{i \in \omega} I_{i}$ is a cut of $M$, and $\left(\bar{I},\left\{A_{0}, \ldots, A_{i}\right\} \mid \bar{I}\right)$ is a $\Sigma_{n}^{0}$-elementary substructure of $\left(I_{i},\left\{A_{0}, \ldots, A_{i}\right\} \mid I_{i}\right)$.

Proof of the claim. Clearly $\bar{I}$ forms a cut, and thus it is always a $\Sigma_{0}^{0}$-elementary substructure. We show $\Sigma_{n}^{0}$-elementarity by induction on the complexity of formulas. Let $0<k \leq n$ and assume that $\left(I_{i},\left\{A_{0}, \ldots, A_{i}\right\}\left\lceil I_{i}\right) \models \exists x \psi(x, c)\right.$ where $c \in \bar{I}$ and $\psi$ is $\Pi_{k-1}^{0}$. By I $\Sigma_{n}^{0}$ in $\left(I_{i},\left\{A_{0}, \ldots, A_{i}\right\}\left\lceil I_{i}\right)\right.$, take the least $d \in I_{i}$ such that $\psi(d, c)$ holds. If $d \notin \bar{I}$, then there exists $j>i$ such that $d \notin I_{j}$, thus $\left(I_{j},\left\{A_{0}, \ldots, A_{i}\right\} \mid I_{j}\right) \models \neg \exists x \psi(x, c)$. This contradicts the second condition of the construction of $I_{i}$ 's, thus $d \in \bar{I}$. Hence, $\left(\bar{I},\left\{A_{0}, \ldots, A_{i}\right\}\lceil\bar{I}) \models \psi(d, c)\right.$ by the induction hypothesis.

Put $\bar{S}=\Delta_{1}^{0}-\operatorname{Def}\left(\bar{I},\left\{A_{i} \mid i \in \omega\right\} \mid \bar{I}\right)$. Then, by the claim and Theorem 1.7, $(\bar{I}, \bar{S}) \models \mathrm{RCA}_{0}+$ $\mathrm{B} \Sigma_{n+1}^{0}$. By the third condition of the construction of $A_{i}$ 's and the $\Sigma_{n}^{0}$-elementarity, we have $(\bar{I}, \bar{S}) \models \Gamma+\neg \varphi(A, a)$. Thus, $\mathrm{RCA}_{0}+\mathrm{B} \Sigma_{n+1}^{0}+\Gamma \nvdash \varphi_{0}$.

Now, we apply Theorem 6.1 to $\mathrm{SGP}_{2}^{2}$. For this, we reformulate the low solution construction for $\mathrm{SGP}_{2}^{2}$ as follows.

Lemma 6.3. Let $\left(M, S_{0}\right)$ be a countable model of $\mathrm{B} \Sigma_{2}^{0}$, and let $f:[\mathbb{N}]^{2} \rightarrow 2$ and $\mathrm{L} \subseteq[\mathbb{N}]^{<\mathbb{N}}$ be members of $S_{0}$. Then, there exists $G \subseteq M$ such that

$$
\begin{aligned}
& \left(M, S_{0} \cup\{G\}\right) \models \mathrm{I} \Sigma_{1}^{0}+\text { " } G \text { is a witness that } \mathrm{L} \text { is not a largeness notion", or, } \\
& \left(M, S_{0} \cup\{G\}\right) \models \mathrm{I} \Sigma_{1}^{0}+\text { " } G \text { is an infinite } \mathrm{L} \text {-grouping for } A_{f}=\left\{x \in \mathbb{N} \mid \lim _{y \rightarrow \infty} f(x, y)=1\right\} \text { ". }
\end{aligned}
$$

Proof. Let $\left(M, S_{0}\right)$ be a countable model of B $\Sigma_{2}^{0}$ such that $f, \mathrm{~L} \in S_{0}$. By Hájek[26], we can always find an w-extension $S \supseteq S_{0}$ such that $(M, S) \models \mathrm{B} \Sigma_{2}^{0}+\mathrm{WKL}_{0}$ (see also Belanger[5]). Thus, we will work on $(M, S)$ instead of $\left(M, S_{0}\right)$. If $\mathrm{L}$ is not a largeness notion in $(M, S)$, take a witness $G \in S$ of not being a largeness notion, then, we have done. Otherwise, we will construct an infinite L-grouping for $A_{f}$.

The following construction is a "model-theoretic interpretation" of Theorem 5.2. To simplify the coding, we will only consider a minimal L-sequence, namely, a sequence of the form $\left\langle F_{i} \in\right.$ $\mathrm{L}|i<k\rangle, F_{i} \subseteq X_{0}, F_{i}<F_{j}$ if $i<j$ and $F_{i} \backslash\left\{\max F_{i}\right\} \notin \mathrm{L}$, i.e., each $F_{i}$ is minimal. A code for a minimal L-sequence is a binary sequence $\sigma \in 2^{<M}$ (which is coded in $M$ ) such that $\{x<|\sigma| \mid \sigma(x)=1\}=\bigcup_{i<k} F_{i}$ for some minimal L-sequence $\left\langle F_{i} \mid i<k\right\rangle$. (By the minimality, one can effectively decode a binary sequence to obtain the L-sequence.) Note that $\sigma$ may code extra 0 's after $\max F_{k-1}$. Thus, one can identify a code $\sigma$ with a pair $\left(\left\langle F_{i} \mid i<k\right\rangle, d\right)$ where $d=|\sigma|$. With this identification, we let $\|\sigma\|_{\mathrm{L}}=k$. Given an (M-)finite sequence of sets $\vec{Y}=\left\langle Y_{j} \mid j<l\right\rangle \in S$ and an $\left(M\right.$-)finite set $F, F$ is said to be consistent with $\vec{Y}$ if for any $j<l,\left(F \subseteq Y_{j} \vee F \subseteq \overline{Y_{j}}\right)$. A code $\sigma$ for a minimal L-sequence $\left\langle F_{i} \mid i<k\right\rangle$ is said to be consistent with $\vec{Y}$ if for any $i<k, F_{i}$ is 
consistent with $\vec{Y}$. Given $t \in M$, we let $A_{f, t}=\left\{x<t \mid x \in X_{0}, \lim _{y \rightarrow \infty, y \in X_{0}} f(x, y)=1\right\}$. Note that $A_{f, t} \in S$ since $(M, S) \models \mathrm{B} \Sigma_{2}^{0}$.

Now, we will construct $G \subseteq M$ by arithmetical forcing. Let $\mathbb{P}$ be the set of all pairs of the form $(\sigma, \vec{Y})$ such that

- $\vec{Y}=\left\langle Y_{j} \mid j<l\right\rangle$ is an (M-)finite sequence of sets in $S$,

- $\sigma$ is a code for a minimal L-sequence which is consistent with $\vec{Y}$ and $A_{f,|\sigma|}$,

and we let $(\sigma, \vec{Y}) \preceq(\tau, \vec{Z})$ if $\sigma \supseteq \tau$ and $\vec{Y} \supseteq \vec{Z}$. Take an $(M, S)$-generic filter $\mathcal{G}$ of $(\mathbb{P}, \preceq)$ and put $G=\bigcup\{\sigma \mid \exists \vec{Y} \in S(\sigma, \vec{Y}) \in \mathcal{G}\}$. Then, this $G$ is the desired. It is clear by construction that $G$ is a minimal L-sequence which is consistent with $A_{f}$. To see that $G$ preserves $I_{1}^{0}$ and $G$ is infinite in $(M, S)$, we need to check the following are dense in $(\mathbb{P}, \preceq)$ :

$\mathcal{D}_{\theta, b}^{1}:=\left\{\begin{array}{c}(\sigma, \vec{Y}) \mid \begin{array}{c}\forall \tau \supseteq \sigma(\tau \text { is a minimal L-sequence consistent with } \vec{Y} \rightarrow \forall n \leq|\tau| \neg \theta(b, n, \tau\lceil n)) \\ \vee \exists c \leq b(\forall d<c \forall \tau \supseteq \sigma(\tau \text { is a minimal L-sequence consistent with } \vec{Y} \\ \rightarrow \forall n \leq|\tau| \neg \theta(d, n, \tau\lceil n)) \wedge \exists n \leq|\sigma| \theta(c, n, \sigma \mid n))\end{array}\end{array}\right\}$,

where $b \in M$ and $\theta(x, n, \sigma) \in \Sigma_{0}^{0}$ with parameters from $(M, S)$,

$\mathcal{D}_{e}^{2}:=\left\{(\sigma, \vec{Y}) \mid\|\sigma\|_{\mathrm{L}} \geq e\right\}$, where $e \in M$.

One can easily see that if $(\sigma, \vec{Y}) \in \mathcal{D}_{\theta, b}^{1}$, then $(\sigma, \vec{Y})$ forces "if $\exists n \theta(b, n, G \mid n)$, there exists least $c \leq b$ such that $\exists n \theta\left(c, n, G\lceil n)\right.$ ", which guarantees $\Sigma_{1}^{0}$-least number principle, and if $(\sigma, \vec{Y}) \in \mathcal{D}_{e}^{2}$, then $(\sigma, \vec{Y})$ forces " $\|G\|_{\mathrm{L}} \geq e$ ", which means $G$ is an infinite minimal L-sequence.

To see that $\mathcal{D}_{\theta, b}^{1}$ is dense, let $(\sigma, \vec{Y})$ be given. Let $\Theta(x)$ be a $\Sigma_{1}^{0}$-formula saying that "there exists $t$ such that for any $\vec{Z}=\left\langle Z_{j} \subseteq[|\sigma|, t)_{\mathbb{N}} \mid j<2^{x}\right\rangle$ there exists $\tau \supseteq \sigma$ with $|\tau| \leq t$ such that $(\tau$ is a minimal L-sequence consistent with $\vec{Y} \frown \vec{Z} \wedge \exists n \leq|\tau| \theta(x, n, \tau\lceil n))$." We consider the two cases.

Case I $\Theta(b)$ fails in $(M, S)$.

In this case, by $\mathrm{WKL}_{0}$ in $(M, S)$, there exists $\vec{Z}=\left\langle Z_{j} \subseteq[|\sigma|, \infty)_{\mathbb{N}} \mid j<2^{b}\right\rangle$ such that $\forall \tau \supseteq \sigma(\tau$ is a minimal L-sequence consistent with $\vec{Y} \neg \vec{Z} \rightarrow \forall n \leq|\tau| \neg \theta(b, n, \tau \mid n))$. Take such $\vec{Z}$. Then, $(\sigma, \vec{Y}-\vec{Z}) \in \mathcal{D}_{\theta, b}^{1}$.

Case II $\Theta(b)$ holds in $(M, S)$.

In this case, by $\mathrm{I} \Sigma_{1}^{0}$ in $(M, S)$, there exists the least $c \leq b$ such that $\Theta(c)$ holds. Then, by $\mathrm{WKL}_{0}$, there exists $\vec{W}^{d}=\left\langle W_{j}^{d} \subseteq[|\sigma|, \infty)_{\mathbb{N}} \mid j<2^{d}\right\rangle$ such that $\forall \tau \supseteq \sigma(\tau$ is a minimal L-sequence consistent with $\left.\vec{Y} \neg \vec{W}^{d} \rightarrow \forall n \leq|\tau| \neg \theta(d, n, \tau \mid n)\right)$ for any $d<c$. Now, take the witness $t \in M$ for $\Theta(c)$, and put $\vec{Z}=\left\langle W_{j}^{d} \cap[|\sigma|, t)_{\mathbb{N}} \mid j<2^{d}, d<c\right\rangle \curlyvee\left\langle A_{f, t} \cap[|\sigma|, t)_{\mathbb{N}}\right\rangle$. Then, by $\Theta(c)$, there exists $\tau \supseteq \sigma$ with $|\tau| \leq t$ such that $\tau$ is a minimal L-sequence consistent with $\vec{Y} \neg \vec{Z}$ and $\exists n \leq|\tau| \theta(c, n, \tau \mid n)$. Then $\left(\tau, \vec{Y} \frown\left\langle W_{j}^{d} \mid j<2^{d}, d<c\right\rangle\right)$ is a condition in $\mathbb{P}$ and $\left(\tau, \vec{Y} \frown\left\langle W_{j}^{d} \mid j<2^{d}, d<c\right\rangle\right) \in \mathcal{D}_{\theta, b}^{1}$.

To see that $\mathcal{D}_{e}^{2}$ is dense, let $(\sigma, \vec{Y})$ be given where $\sigma$ is a code for $\left\langle F_{i} \mid i<k\right\rangle$ and $\vec{Y}=\left\langle Y_{j} \mid j<l\right\rangle$. By applying Lemma $2.1 e$ times in $(M, S)$, one can find $\left\langle t_{0}, \ldots, t_{e}\right\rangle$ such that $t_{0}=|\sigma|$ and for any $s<e$ and for any $2^{l+1}$ splitting $M=\bigsqcup_{p<2^{l+1}} W_{p}$, there exists a finite subset of $\left[t_{s}, t_{s+1}\right)_{\mathbb{N}}$ which is L-large and included in one of $W_{p}$. ( $\left(\Sigma_{1}^{0}\right.$ is enough for this iteration.) Thus, there exists $E_{s} \subseteq\left[t_{s}, t_{s+1}\right)_{\mathbb{N}}$ which is L-large and consistent with $\vec{Y}$ and $A_{f, t_{e}}$ for any $s<e$. Let $\tau \supseteq \sigma$ be a code for a sequence $\left\langle F_{i} \mid i<k\right\rangle \frown\left\langle E_{s} \mid s<e\right\rangle$. Then, $(\tau, \vec{Y})$ is a condition in $\mathbb{P}$ and $\|\tau\|_{\mathrm{L}} \geq e$, thus, $(\tau, \vec{Y}) \in \mathcal{D}_{e}^{2}$. 
Theorem 6.4. $\mathrm{RCA}_{0}+\mathrm{SGP}_{2}^{2}$ is a $\tilde{\Pi}_{3}^{0}$-conservative extension of $\mathrm{I} \Sigma_{1}^{0}$.

Proof. Straightforward from Lemma 5.1, Theorem 6.1 and Lemma 6.3.

Thus, by the amalgamation theorem, we have the following conservation result.

Theorem 6.5. $\mathrm{WKL}_{0}+\mathrm{GP}_{2}^{2}+\mathrm{ADS}$ is a $\tilde{\Pi}_{3}^{0}$-conservative extension of $\mathrm{I} \Sigma_{1}^{0}$.

Proof. Apply Theorem 3.6 for the conservation results Corollary 4.5 and Theorem 6.4, then we can see that $\mathrm{WKL}_{0}+\mathrm{ADS}+\mathrm{SGP}_{2}^{2}$ is a $\tilde{\Pi}_{3}^{0}$ conservative extension of $\mathrm{I} \Sigma_{1}^{0}$. By Hirschfeldt and Shore [32], ADS implies $\mathrm{COH}$. Thus, $\mathrm{WKL}_{0}+\mathrm{GP}_{2}^{2}+\mathrm{ADS}$ is a $\tilde{\Pi}_{3}^{0}$-conservative extension of $\mathrm{I} \Sigma_{1}^{0}$.

\section{Conservation theorem for $\mathrm{RT}_{2}^{2}$}

In this section, we will show that $\mathrm{WKL}_{0}+\mathrm{RT}_{2}^{2}$ is a $\tilde{\Pi}_{3}^{0}$-conservative extension of $\mathrm{I} \Sigma_{1}^{0}$ by showing that $\mathrm{WKL} L_{0}+\mathrm{EM}$ is a $\tilde{\Pi}_{3}^{0}$-conservative extension of $\mathrm{I} \Sigma_{1}^{0}$. For this, we will bound the size of $\omega^{k}$-large(EM) sets by $\omega^{n}$-large sets using the following finite grouping principle.

Definition 7.1 ( $\mathrm{I} \Sigma_{1}^{0}$, finite grouping principle). Given largeness notions $\mathrm{L}_{1}, \mathrm{~L}_{2}$ and a coloring $f:[X]^{n} \rightarrow k,\left(\mathrm{~L}_{1}, \mathrm{~L}_{2}\right)$-grouping for $f$ is a finite family of finite sets $\left\langle F_{i} \subseteq X \mid i<l\right\rangle$ such that

- $\forall i<j<l F_{i}<F_{j}$,

- $\forall i<l F_{i} \in \mathrm{L}_{1}$,

- for any $H \subseteq$ fin $\mathbb{N}$, if $H \cap F_{i} \neq \emptyset$ for any $i<l$, then $H \in \mathrm{L}_{2}$, and,

- $\forall i_{1}<\cdots<i_{n} \exists c<k \forall x_{1} \in F_{i_{1}}, \ldots, \forall x_{n} \in F_{i_{n}} f\left(x_{1}, \ldots, x_{n}\right)=c$.

Note that if $\mathrm{L}_{2}$ is regular, then the third condition can be replaced with $\left\{\max F_{i} \mid i<l\right\} \in \mathrm{L}_{2}$. Now $\operatorname{FGP}_{k}^{n}\left(\mathrm{~L}_{1}, \mathrm{~L}_{2}\right)$ (finite grouping principle for $\left(\mathrm{L}_{1}, \mathrm{~L}_{2}\right)$ ) asserts that for any infinite set $X_{0} \subseteq \mathbb{N}$, there exists a finite set $X \subseteq X_{0}$ such that for any coloring $f:[X]^{n} \rightarrow k$, there exists a $\left(\mathrm{L}_{1}, \mathrm{~L}_{2}\right)$-grouping for $f$.

Theorem 7.1. Let $\mathrm{L}_{1}$ and $\mathrm{L}_{2}$ be $\Delta_{0}$-definable regular largeness notions provably in $\mathrm{I} \Sigma_{1}^{0}$. Then, $\mathrm{I} \Sigma_{1}^{0}$ proves $\mathrm{FGP}_{2}^{2}\left(\mathrm{~L}_{1}, \mathrm{~L}_{2}\right)$.

Proof. One can easily check that $\mathrm{FGP}_{2}^{2}\left(\mathrm{~L}_{1}, \mathrm{~L}_{2}\right)$ is a $\tilde{\Pi}_{3}^{0}$-statement which is provable from $\mathrm{WKL}_{0}+$ $\mathrm{GP}_{2}^{2}\left(\mathrm{~L}_{1}\right)$. Thus, $\mathrm{I}_{1}^{0}$ proves $\mathrm{FGP}_{2}^{2}\left(\mathrm{~L}_{1}, \mathrm{~L}_{2}\right)$ by Theorem 6.5 .

Now we apply the generalized Parsons theorem to the finite grouping principle. Actually, its upper bound also bounds $\omega^{k}$-large(EM) sets as follows.

Lemma 7.2. For any $k \in \omega$, there exists $n \in \omega$ such that

$$
\mathrm{I} \Sigma_{1}^{0} \vdash \forall Z \subseteq_{\text {fin }}(3, \infty)_{\mathbb{N}}\left(Z \text { is } \omega^{n} \text {-large } \rightarrow Z \text { is } \omega^{k} \text {-large }(\mathrm{EM})\right) \text {. }
$$

Proof. We will prove this by (external) induction. For the case $k=1, n=6$ is enough by Theorem 2.6. Assume now $k>1$ and $\omega^{n_{0}}$-largeness implies $\omega^{k-1}$-large(EM)ness in $\mathrm{I} \Sigma_{1}^{0}$. By Corollary 3.5 and Theorem 7.1, take $n \in \omega$ so that $\mathrm{I} \Sigma_{1}^{0}$ proves $\forall Z \subseteq$ fin $\mathbb{N}\left(Z\right.$ is $\omega^{n}$-large $\rightarrow$ any coloring $f:[Z]^{2} \rightarrow 2$ has an $\left(\omega^{n_{0}}, \omega^{6}\right)$-grouping). Within $\mathrm{I} \Sigma_{1}^{0}$, given an $\omega^{n}$-large set $Z \subseteq(3, \infty)_{\mathbb{N}}$ and $f:[Z]^{2} \rightarrow 2$, we want to find $H \subseteq Z$ such that $f$ is transitive on $[H]^{2}$ and $H$ is $\omega^{k}$-large. By the assumption, there exists an $\left(\omega^{n_{0}}, \omega^{6}\right)$-grouping $\left\langle F_{i} \mid i<l\right\rangle \subseteq Z$ for $f$. Since each $F_{i}$ 
is $\omega^{n_{0}}$-large, it is $\omega^{k-1}$-large(EM), thus, there exists $H_{i} \subseteq F_{i}$ such that $H_{i}$ is $\omega^{k-1}$-large and $f$ is transitive on $\left[H_{i}\right]^{2}$. On the other hand, $\left\{\max F_{i} \mid i<l\right\}$ is $\omega^{6}$-large, thus, there exists $\tilde{H} \subseteq\left\{\max F_{i} \mid i<l\right\}$ such that $\tilde{H}$ is $\omega$-large and $f$ is constant on $[\tilde{H}]^{2}$ by Theorem 2.6. Put $H=\bigcup\left\{H_{i} \mid i<l, \max F_{i} \in \tilde{H}\right\}$. Then, one can easily check that $H$ is $\omega^{k}$-large. We now show that $f$ is transitive on $[H]^{2}$. Let $a, b, c \in H$ and $a<b<c$. If there exists $i<l$ such that $a, b, c \in H_{i}$, then $f$ is transitive for $a, b, c$ since $f$ is transitive on $\left[H_{i}\right]^{2}$. If for some $i_{0}<i_{1}<l, a, b \in H_{i_{0}}$ and $c \in H_{i_{1}}$, then, $f(a, c)=f(b, c)$, so $f$ is transitive for $a, b, c$. The case $a \in H_{i_{0}}$ and $b, c \in H_{i_{1}}$ for some $i_{0}<i_{1}<l$ is similar. Finally, if for some $i_{0}<i_{1}<i_{2}<l, a \in H_{i_{0}}, b \in H_{i_{1}}$ and $c \in H_{i_{2}}$, then $f(a, b)=f\left(\max F_{i_{0}}, \max F_{i_{1}}\right)=f\left(\max F_{i_{0}}, \max F_{i_{2}}\right)=f(a, c)$, thus $f$ is transitive for $a, b, c$.

Theorem 7.3. $\mathrm{WKL}_{0}+\mathrm{EM}$ is a $\tilde{\Pi}_{3}^{0}$-conservative extension of $\mathrm{I} \Sigma_{1}^{0}$.

Proof. By Theorems 2.4, 3.1 and Lemma 7.2.

Now the main theorem follows from the amalgamation theorem.

Theorem 7.4. $\mathrm{WKL}_{0}+\mathrm{RT}_{2}^{2}$ is a $\tilde{\Pi}_{3}^{0}$-conservative extension of $\mathrm{I} \Sigma_{1}^{0}$.

Proof. By Theorem 3.6, Corollary 4.5 and Theorem 7.3.

Seetapun and Slaman [55] asked whether $\mathrm{RCA}_{0}+\mathrm{RT}_{2}^{2}$ proves the consistency of $\mathrm{I} \Sigma_{1}^{0}$, and Cholak, Jockusch and Slaman [13] asked whether $\mathrm{RCA}_{0}+\mathrm{RT}_{2}^{2}$ proves the totality of Ackermann function. We answer negatively through the main theorem.

Corollary 7.5. $\mathrm{WKL}_{0}+\mathrm{RT}_{2}^{2}$ does not imply the consistency of $\mathrm{I} \Sigma_{1}^{0}$ nor the totality of Ackermann function.

Chong and Yang [17] asked what the proof-theoretic ordinal of $\mathrm{RCA}_{0}+\mathrm{RT}_{2}^{2}$ is. We again answer this question through the main theorem.

Corollary 7.6. The proof-theoretic ordinal of $\mathrm{RCA}_{0}+\mathrm{RT}_{2}^{2}$ or $\mathrm{WKL}_{0}+\mathrm{RT}_{2}^{2}$ is $\omega^{\omega}$.

Note that one can avoid using the amalgamation theorem by directly combining the bounds for large $\left(\mathrm{psRT}_{2}^{2}\right)$ ness and large(EM)ness in order to obtain a bound for $\omega^{k}$-large $\left(\mathrm{RT}_{2}^{2}\right)$ sets and reprove the main conservation theorem.

Proposition 7.7. For any $k \in \omega$, there exists $n \in \omega$ such that

$$
\mathrm{I} \Sigma_{1}^{0} \vdash \forall Z \subseteq_{\text {fin }}(3, \infty)_{\mathbb{N}}\left(Z \text { is } \omega^{n} \text {-large } \rightarrow Z \text { is } \omega^{k} \text {-large }\left(\mathrm{RT}_{2}^{2}\right)\right) .
$$

Proof. Given $k \in \omega$, take $n \in \omega$ so that $\mathrm{I} \Sigma_{1}^{0}$ proves $\forall Z \subseteq_{\text {fin }}(3, \infty)_{\mathbb{N}}\left(Z\right.$ is $\omega^{n}$-large $\rightarrow Z$ is $\omega^{2 k+6}$-large $\left.(\mathrm{EM})\right)$ by Lemma 7.2 . Then, within $\mathrm{I} \Sigma_{1}^{0}$, given $Z \subseteq_{\text {fin }}(3, \infty)_{\mathbb{N}}$ which is $\omega^{n}$-large and $f:[Z]^{2} \rightarrow 2$, there exists $H_{0} \subseteq Z$ such that $H_{0}$ is $\omega^{2 k+6}$-large and $f$ is transitive on $\left[H_{0}\right]^{2}$. Then, by Lemma 4.4, there exists $H \subseteq H_{0}$ such that $H$ is $\omega^{k}$-large and $f$ is constant on $[H]^{2}$. Thus, $Z$ is $\omega^{k}$-large $\left(\mathrm{RT}_{2}^{2}\right)$.

Then, Theorem 7.4 follows from Theorems 2.4, 3.1 and Proposition 7.7. 


\section{Formalizing the conservation proof}

In this section, we will formalize Theorem 7.4 within PRA. Actually, most arguments we used are straightforwardly formalizable within $\mathrm{WKL}_{0}$. We however need to take care of the use of external induction and non-computable construction of models. We fix a standard provability predicate $\vdash$.

Lemma 8.1. The following are provable within $\mathrm{WKL}_{0}$.

(1) $\forall \varphi \in \Pi_{1}^{1}\left(\left(\mathrm{WKL}_{0} \vdash \varphi\right) \rightarrow\left(\mathrm{I} \Sigma_{1}^{0} \vdash \varphi\right)\right)($ Theorem 1.3).

(2) $\forall n \in \mathbb{N}\left(\mathrm{I} \Sigma_{1}^{0} \vdash\right.$ any infinite set has an $\omega^{n}$-large subset) (Theorem 2.4).

(3) $\forall k \in \mathbb{N}\left(\mathrm{I} \Sigma_{1}^{0} \vdash X\right.$ is $\omega^{k+4}$-large $\wedge \min X>3 \rightarrow X$ is $\omega$-large $\left.\left(\mathrm{RT}_{k}^{2}\right)\right)$ (Theorem 2.6).

(4) The generalized Parsons theorem (Corollary 3.5).

(5) The amalgamation theorem (Theorem 3.6).

(6) $\left(\forall n \in \mathbb{N}\left(\mathrm{I} \Sigma_{1}^{0} \vdash\right.\right.$ any infinite set has an $\omega^{n}$-large $(\Gamma)$ subset $\left.)\right) \rightarrow\left(\forall \varphi \in \tilde{\Pi}_{3}^{0}\left(\left(\mathrm{WKL}_{0}+\Gamma \vdash \varphi\right) \rightarrow\right.\right.$ $\left.\left.\left(\mathrm{I} \Sigma_{1}^{0} \vdash \varphi\right)\right)\right)$ for $\Gamma \equiv \mathrm{psRT}_{2}^{2}$, EM (Theorem 3.1).

(7) $\forall n \in \mathbb{N}\left(I_{1}^{0} \vdash\right.$ any infinite set has an $\omega^{n}$-large $\left(\mathrm{psRT}_{2}^{2}\right)$ subset) (Lemma 4.4).

(8) $\forall \varphi \in \tilde{\Pi}_{3}^{0}\left(\left(\mathrm{WKL}_{0}+\mathrm{GP}_{2}^{2} \vdash \varphi\right) \rightarrow\left(\mathrm{I} \Sigma_{1}^{0} \vdash \varphi\right)\right)($ Theorem 6.5).

(9) $\forall k \in \mathbb{N}\left(\mathrm{I \Sigma}_{1}^{0} \vdash \mathrm{FGP}_{2}^{2}\left(\mathrm{~L}_{\omega^{k}}, \mathrm{~L}_{\omega^{6}}\right)\right)$ (Theorem \%.1).

(10) $\forall n \in \mathbb{N}\left(\mathrm{I} \Sigma_{1}^{0} \vdash\right.$ any infinite set has an $\omega^{n}$-large(EM) subset) (Lemma \%.2).

Proof. We reason within WKL $\mathrm{W}_{0}$. For (1), several formalized proofs are known. See, e.g., [3, 26]. For (2), the induction used here is on provability, thus it is a $\Sigma_{1}^{0}$-induction. For (3), the original Ketonen and Solovay's proof is directly formalizable (see [37, Section 6]). For (4) and (5), we can directly formalize our model-theoretic proofs of Corollary 3.5 and Theorem 3.6 by using the completeness theorem which is available within $\mathrm{WKL}_{0}$. For (6), we can formalize the proofs of Lemma 3.2 and Theorem 3.3 by using the completeness theorem. To formalize the proof of Theorem 3.1, we use the induction on provability. For (7), formalize the proof of Lemma 4.4. Formalization is direct since we only deal with finite objects. For (8), formalize the argument in Section 6. To formalize the proof of Theorem 6.1, an $\omega$-extension to be a model of $B \Sigma_{2}^{0}+W K L_{0}$ is available within $W K L_{0}$ by formalizing the argument by Hájek[26] or Belanger[5]. The existence of a countable recursively saturated model is provable in $\mathrm{WKL}_{0}$ (see [57, Section IX]) and the Theorem 6.2 can be formalized similarly. To formalize the proof of Lemma 6.3, one can take a generic by the Baire category theorem which is available within $\mathrm{RCA}_{0}$. (9) is straightforward from (8). For (10), formalize the proof of Lemma 7.2. The induction used here is again on provability.

Thus, we have the following formalized conservation theorem.

Theorem 8.2. PRA proves that $\mathrm{WKL}_{0}+\mathrm{RT}_{2}^{2} \vdash \psi$ implies $\mathrm{I} \Sigma_{1}^{0} \vdash \psi$ for any $\tilde{\Pi}_{3}^{0}$-sentences.

Now the consistency equivalence of $\mathrm{I} \Sigma_{1}^{0}$ and $\mathrm{WK} \mathrm{L}_{0}+\mathrm{RT}_{2}^{2}$ follows from this formalized conservation theorem.

Corollary 8.3. Over PRA, $\operatorname{Con}\left(\mathrm{I} \Sigma_{1}^{0}\right)$ is equivalent to $\operatorname{Con}\left(\mathrm{WKL}_{0}+\mathrm{RT}_{2}^{2}\right)$. 


\section{Open questions}

In their paper [14], Chong, Slaman and Yang asked whether $\mathrm{RT}_{2}^{2}$ is a $\Pi_{1}^{1}$-conservative extension of $\mathrm{RCA}_{0}+\mathrm{B} \Sigma_{2}^{0}$. This question remains open, and a positive answer would strengthen our main conservation result since $\mathrm{B} \Sigma_{2}^{0}$ is $\Pi_{3}^{0}$-conservative over $\mathrm{I} \Sigma_{1}^{0}$ (Theorem 1.5).

Question 9.1 (Chong, Slaman, Yang). Is $\mathrm{RT}_{2}^{2}$ a $\Pi_{1}^{1}$-conservative extension of $\mathrm{RCA}+\mathrm{B} \Sigma_{2}^{0}$ ?

In particular, they proved [16] that the chain anti-chain principle is $\Pi_{1}^{1}$-conservative over $\mathrm{RCA}_{0}+$ $\mathrm{B} \Sigma_{2}^{0}$. Therefore, in order to answer Question 9.1, one needs only to prove that this is also the case for the Erdős-Moser theorem.

For the purposes of our conservation proof, we introduced the grouping principle, which seems to be interesting to study in its own right. First, what is the first-order strength of $\mathrm{GP}_{2}^{2}$ ? Alexander Kreuzer [40] gave a partial answer to this question by proving the following theorem.

Theorem 9.1 (Kreuzer). Over $\mathrm{RCA}_{0}, \mathrm{SGP}_{2}^{2}$ implies $\mathrm{B} \Sigma_{2}^{0}$.

Proof. Assume that $\mathrm{B} \Sigma_{2}^{0}$ fails. As in Remark 2.2, there exist a partition $X=X_{0} \sqcup \cdots \sqcup X_{k-1}$ such that each of the $X_{i}$ 's is finite, and then $\mathrm{L}=\left\{F \in[\mathbb{N}]^{<\mathbb{N}} \mid \forall i<k\left(F \nsubseteq X_{i}\right)\right\}$ is a largeness notion. Define $f:[\mathbb{N}]^{2} \rightarrow 2$ as $f(x, y)=1 \leftrightarrow \exists i<k\left(x \in X_{i} \wedge y \in X_{i}\right)$. Since $X_{i}$ 's are all finite, $f$ is a stable coloring. By $\mathrm{SGP}_{2}^{2}$, there exists an infinite L-grouping $\left\langle F_{j} \mid j \in \mathbb{N}\right\rangle$. Since each of $F_{j}$ is not included in any of $X_{i}$ 's, for any $j<j^{\prime}$, the color between $F_{j}$ and $F_{j^{\prime}}$ is 0 . Thus, $\min F_{0}, \ldots, \min F_{k}$ are in different $X_{i}$ 's, which is a contradiction.

Still, the following questions are remained open.

Question 9.2. For some $k \in \omega$, does $\mathrm{GP}_{2}^{2}\left(\mathrm{~L}_{\omega^{k}}\right)$ imply $\mathrm{B} \Sigma_{2}^{0}$ over $\mathrm{RCA}_{0}$ ?

Question 9.3. Is $\mathrm{GP}_{2}^{2}$ a $\Pi_{1}^{1}$-conservative extension of $\mathrm{RCA}_{0}+\mathrm{B} \Sigma_{2}^{0}$ ?

The grouping principle has been used to establish a density bound for the Erdős-Moser theorem. The stable grouping principle does not imply the stable version of the Erdős-Moser theorem since the former admits low solutions whereas the latter does not. It is however unknown whether the full version of the two principles coincide.

Question 9.4. Does EM imply $\mathrm{GP}_{2}^{2}$ over $\mathrm{RCA}_{0}$ ?

Our conservation proof contains almost no information about the size of the proof, but it is interesting to know whether $\mathrm{RT}_{2}^{2}$ gives shorter proofs for $\tilde{\Pi}_{3}^{0}$-consequences of $\mathrm{I} \Sigma_{1}^{0}$ or not.

Question 9.5. Does $\mathrm{WKL}_{0}+\mathrm{RT}_{2}^{2}$ or $\mathrm{WKL}_{0}+\mathrm{psRT}_{2}^{2}$ have a significant speed-up over $\mathrm{I} \Sigma_{1}^{0}$ ?

Note that there is no significant speed-up between $\mathrm{WKL}_{0}$ and $\mathrm{I} \Sigma_{1}^{0}$ (see Avigad[3]). A killer example for this question is an existence of $m$-dense sets, i.e., $m-\widetilde{\mathrm{PH}}\left(\mathrm{RT}_{2}^{2}\right)$ or $m$ - $\widetilde{\mathrm{PH}}\left(\mathrm{psRT}_{2}^{2}\right)$. It is not hard to see that $m$ - $\widetilde{\mathrm{PH}}\left(\mathrm{RT}_{2}^{2}\right)$ can be proved from $\mathrm{WKL}_{0}+\mathrm{RT}_{2}^{2}$ by using $\mathrm{RT}_{2}^{2} m$-times, and the case of $\mathrm{psRT}_{2}^{2}$ is similar. On the other hand, to prove $m-\widetilde{\mathrm{PH}}\left(\mathrm{psRT}_{2}^{2}\right)$ from $\mathrm{I} \Sigma_{1}^{0}$, what we know is the following.

Proposition 9.2. For any $m \in \omega$, the following is provable within $\mathrm{I} \Sigma_{1}^{0}$. If a finite set $X \subseteq \mathbb{N}$ is $\omega^{3^{m+1}}$-large and $\min X>3$, then $X$ is $m$-dense $\left(\mathrm{psRT}_{2}^{2}\right)$.

Proof. Easy induction by using Lemma 4.4. 
Thus, within $\mathrm{I} \Sigma_{1}^{0}$, we can obtain an $m$-dense(psRT $\left.\mathrm{pr}_{2}^{2}\right)$-set by using $\Sigma_{1}^{0}$-induction $3^{m+1}$-times. This might indicate that there is at most exponential speed-up for the case of $\mathrm{psRT}_{2}^{2}$. For the case of $\mathrm{RT}_{2}^{2}$, the situation is more difficult. An only upper bound for $m$-dense $\left(\mathrm{RT}_{2}^{2}\right)$ sets obtained from our argument is the following.

Proposition 9.3. There exists a primitive recursive function $h: \omega \rightarrow \omega$ such that for any $m \in \omega$, the following is provable within $\mathrm{I} \Sigma_{1}^{0}$. If a finite set $X \subseteq \omega$ is $\omega^{h(m)}$-large then $X$ is $m$-dense $\left(\mathrm{RT}_{2}^{2}\right)$.

Proof. By formalizing the proof of Proposition 7.7 and applying $\Sigma_{1}^{0}$-induction, we obtain

$$
\forall m \in \mathbb{N} \exists k \in \mathbb{N}\left(\mathrm{I} \Sigma_{1}^{0} \vdash X \text { is } \omega^{k} \text {-large } \rightarrow X \text { is } m \text {-dense }\left(\mathrm{RT}_{2}^{2}\right)\right) .
$$

Then, by the Parsons theorem, there exists a primitive recursive function $h: \omega \rightarrow \omega$ such that $\mathrm{I} \Sigma_{1}^{0}$ proves

$$
\forall m \in \mathbb{N} \exists k \leq h(m)\left(\mathrm{I}_{1}^{0} \vdash_{h(m)} X \text { is } \omega^{k} \text {-large } \rightarrow X \text { is } m \text {-dense }\left(\mathrm{RT}_{2}^{2}\right)\right),
$$

where $\vdash_{x}$ means that there exists a proof whose Gödel number is smaller than $x$. For $m \in \omega, k$ and $h(m)$ in the above are standard, thus, I $\Sigma_{1}^{0}$ truly proves " $X$ is $\omega^{k}$-large $\rightarrow X$ is $m$-dense $\left(\mathrm{RT}_{2}^{2}\right)$ ".

\section{References}

[1] Peter Aczel. Two notes on the Paris independence result. In Model theory and arithmetic (Paris, 1979-1980), volume 890 of Lecture Notes in Math., pages 21-31. Springer, Berlin-New York, 1981.

[2] Spiros A. Argyros and Stevo Todorcevic. Ramsey methods in analysis. Advanced Courses in Mathematics. CRM Barcelona. Birkhäuser Verlag, Basel, 2005.

[3] Jeremy Avigad. Formalizing forcing arguments in subsystems of second-order arithmetic. Annals of Pure and Applied Logic, 82:165-191, 1996.

[4] John Barwise and John Schlipf. An introduction to recursively saturated and resplendent models. Journal of Symbolic Logic, 41(2):531-536, 1976.

[5] David A. Belanger. Conservation theorems for the cohesiveness principle, 2015. To appear. Available at http://www.math.nus.edu.sg/ imsdrb/papers/coh-2015-09-30.pdf.

[6] Paul Benacerraf and Hilary Putnam, editors. Philosophy of mathematics. Selected readings. Cambridge University Press, second edition, 1983. viii+600 pages.

[7] Teresa Bigorajska and Henryk Kotlarski. A partition theorem for $\alpha$-large sets. Fund. Math., 160(1):27-37, 1999.

[8] Teresa Bigorajska and Henryk Kotlarski. Some combinatorics involving $\xi$-large sets. Fund. Math., 175(2):119-125, 2002.

[9] Teresa Bigorajska and Henryk Kotlarski. Partitioning $\alpha$-large sets: some lower bounds. Trans. Amer. Math. Soc., 358(11):4981-5001, 2006.

[10] Andrey Bovykin and Andreas Weiermann. The strength of infinitary Ramseyan principles can be accessed by their densities. accepted for publication in Ann. Pure Appl. Logic, http: //logic.pdmi.ras.ru/ andrey/research.html, 2005. 
[11] Wilfried Buchholz. Proof-theoretic analysis of termination proofs. Annals of Pure and Applied Logic, 75:57-65, 1995.

[12] Samuel R. Buss, editor. Handbook of Proof Theory, volume 137 of Studies in Logic and the Foundations of Mathematics. North-Holland, 1998.

[13] Peter A. Cholak, Carl G. Jockusch, and Theodore A. Slaman. On the strength of Ramsey's theorem for pairs. The Journal of Symbolic Logic, 66(1):1-15, 2001.

[14] C. T. Chong, Theodore A. Slaman, and Yue Yang. The inductive strength of Ramsey's theorem for pairs, 2014. Preprint.

[15] C. T. Chong, Theodore A. Slaman, and Yue Yang. The metamathematics of Stable Ramsey's Theorem for Pairs. J. Amer. Math. Soc., 27(3):863-892, 2014.

[16] Chi-Tat Chong, Theodore A. Slaman, and Yue Yang. $\Pi_{1}^{1}$-conservation of combinatorial principles weaker than Ramsey's Theorem for pairs. Advances in Matheamtics, 230:1060-1077, 2012.

[17] Chi Tat Chong and Yue Yang. Ramsey's theorem, reverse mathematics and nonstandard models. Asia Pac. Math. Newsl., 5(2):1-4, 2015.

[18] P. Clote. Partition relations in arithmetic. In Methods in mathematical logic (Caracas, 1983), volume 1130 of Lecture Notes in Math., pages 32-68. Springer, Berlin, 1985.

[19] Chris J. Conidis and Theodore A. Slaman. Random reals, the rainbow Ramsey theorem, and arithmetic conservation. Journal of Symbolic Logic, 78(1):195-206, 2013.

[20] Rod Downey, Denis R. Hirschfeldt, Steffen Lempp, and Reed Solomon. A $\Delta_{2}^{0}$ set with no infinite low subset in either it or its complement. Journal of Symbolic Logic, pages 1371-1381, 2001.

[21] P. Erdös. Some remarks on the theory of graphs. Bull. Amer. Math. Soc., 53:292-294, 1947.

[22] Stephen Flood. Reverse mathematics and a Ramsey-type König's lemma. Journal of Symbolic Logic, 77(4):1272-1280, 2012.

[23] H. Friedman. Personal communication to L. Harrington, 1977.

[24] Kurt Gödel. Über formal unentscheidbare Sätze der principia mathematica und verwandter Systeme. I. Monatsh. Math., 149(1):1-30, 2006. Reprinted from Monatsh. Math. Phys. 38 (1931), 173-198 [MR1549910], With an introduction by Sy-David Friedman.

[25] Ronald L. Graham, Bruce L. Rothschild, and Joel H. Spencer. Ramsey theory. Wiley Series in Discrete Mathematics and Optimization. John Wiley \& Sons, Inc., Hoboken, NJ, 2013. Paperback edition of the second (1990) edition.

[26] Petr Hájek. Interpretability and fragments of arithmetic. In P. Clote and J. Krajíček, editors, Arithmetic, Proof Theory and Computational Complexity, pages 185-196. Oxford, Clarendon Press, 1993.

[27] Petr Hájek and Pavel Pudlák. Metamathematics of First-Order Arithmetic. Springer-Verlag, Berlin, 1993. XIV+460 pages. 
[28] John M. Harris, Jeffry L. Hirst, and Michael J. Mossinghoff. Combinatorics and graph theory. Undergraduate Texts in Mathematics. Springer, New York, second edition, 2008.

[29] David Hilbert. Über das Unendliche. Mathematische Annalen, 95:161-190, 1926. English translation in $[61,6]$.

[30] David Hilbert and Paul Bernays. Grundlagen der Mathematik. I/Foundations of mathematics. I. Part A. Prefaces and $\S \S 1-2$. College Publications, London, 2011. Edited and with a preface by Dov Gabbay, Michael Gabbay, Jörg Siekmann and Claus-Peter Wirth, Commented translation by Claus-Peter Wirth of the second German edition of 1968, including the annotation and translation of all deleted parts of the first German edition of 1934, With a chapter "Hilbert's proof theory" by Wilfried Sieg [MR2668182], Dual German-English text.

[31] Denis R Hirschfeldt. Slicing the truth. Lecture Notes Series, Institute for Mathematical Sciences, National University of Singapore, 28, 2014.

[32] Denis R. Hirschfeldt and Richard A. Shore. Combinatorial principles weaker than Ramsey's theorem for pairs. Journal of Symbolic Logic, 72:171-206, 2007.

[33] Jeffry Lynn Hirst. Combinatorics in Subsystems of Second Order Arithmetic. PhD thesis, The Pennsylvania State University, August 1987.

[34] Carl G Jockusch. Ramsey's theorem and recursion theory. Journal of Symbolic Logic, 37(2):268-280, 1972.

[35] Carl G Jockusch and Robert I Soare. $\Pi_{1}^{0}$ classes and degrees of theories. Transactions of the American Mathematical Society, 173:33-56, 1972.

[36] R. Kaye. Models of Peano Arithmetic. Oxford University Press, 1991.

[37] Jussi Ketonen and Robert Solovay. Rapidly growing Ramsey functions. Ann. of Math. (2), 113(2):267-314, 1981.

[38] Roman Kossak and James H. Schmerl. The structure of models of Peano arithmetic. Oxford Logic Guides, 50. Oxford University Press, Oxford, 2006. XIV+311 pages.

[39] Henryk Kotlarskia, Bożena Piekartb, and Andreas Weiermann. More on lower bounds for partitioning $\alpha$-large sets. Annals of Pure and Applied Logic, 147:113-126, 2007.

[40] Alexander P. Kreuzer. Private communication, January 2016.

[41] Alexander P. Kreuzer. Primitive recursion and the chain antichain principle. Notre Dame Journal of Formal Logic, 53(2):245-265, 2012.

[42] Alexander P. Kreuzer and Ulrich Kohlenbach. Ramsey's theorem for pairs and provably recursive functions. Notre Dame Journal of Formal Logic, 50:427-444, 2009.

[43] Manuel Lerman, Reed Solomon, and Henry Towsner. Separating principles below Ramsey's theorem for pairs. Journal of Mathematical Logic, 13(02):1350007, 2013.

[44] Jiayi Liu. RT 2 does not imply $\mathrm{WKL}_{0}$. Journal of Symbolic Logic, 77(2):609-620, 2012.

[45] Joseph Roy Mileti. Partition theorems and computability theory. PhD thesis, Carnegie Mellon University, 2004. 
[46] Joseph S. Miller. Assorted results in and about effective randomness. In preparation.

[47] Shota Murakami, Takeshi Yamazaki, and Keita Yokoyama. On the ramseyan factorization theorem. In Language, Life, Limits, pages 324-332. Springer, 2014.

[48] J. B. Paris. Some independence results for Peano Arithmetic. Journal of Symbolic Logic, 43(4):725-731, 1978.

[49] J. B. Paris and L. A. S. Kirby. $\Sigma_{n}$-collection schemas in arithmetic. In Logic Colloquium 'ry (Proc. Conf., Wroclaw, 1977), volume 96 of Stud. Logic Foundations Math., pages 199-209, 1978.

[50] Ludovic Patey. Iterative forcing and hyperimmunity in reverse mathematics. In Arnold Beckmann, Victor Mitrana, and Mariya Soskova, editors, Evolving Computability, volume 9136 of Lecture Notes in Computer Science, pages 291-301. Springer International Publishing, 2015.

[51] Ludovic Patey. Somewhere over the rainbow Ramsey theorem for pairs. Submitted. Available at http://arxiv.org/abs/1501.07424, 2015.

[52] Ludovic Patey. The reverse mathematics of Ramsey-type theorems. PhD thesis, Université Paris Diderot, 2016.

[53] Dominique Perrin and Jean-Éric Pin. Infinite Words: Automata, Semigroups, Logic and Games, volume 141 of Pure and Applied Mathematics. Elsevier, 2004.

[54] Andreas Podelski and Andrey Rybalchenko. Transition invariants. In Logic in Computer Science: Modelling and Reasoning about Systems, pages 32-41, 2004.

[55] David Seetapun and Theodore A. Slaman. On the strength of Ramsey's theorem. Notre Dame Journal of Formal Logic, 36(4):570-582, 1995.

[56] Stephen G. Simpson. Partial realizations of Hilbert's program. Journal of Symbolic Logic, 53:349-363, 1988.

[57] Stephen G. Simpson. Subsystems of Second Order Arithmetic. Perspectives in Mathematical Logic. Springer-Verlag, 1999. XIV + 445 pages; Second Edition, Perspectives in Logic, Association for Symbolic Logic, Cambridge University Press, 2009, XVI+ 444 pages.

[58] Michiel De Smet and Andreas Weiermann. Partitioning $\alpha$-large sets for $\alpha<\varepsilon_{\omega}$. Available at http://arxiv.org/abs/1001.2437.

[59] Silvia Steila and Keita Yokoyama. Reverse mathematical bounds for the termination theorem. Submitted. Available at http://arxiv.org/abs/1512.08622.

[60] William W Tait. Finitism. The Journal of Philosophy, pages 524-546, 1981.

[61] Jean van Heijenoort. From Frege to Gödel. A source book in mathematical logic, 1879-1931. Harvard University Press, 1967. xi+660 pages.

[62] Wei Wang. The definability strength of combinatorial principles, 2014. To appear. Available at http://arxiv.org/abs/1408.1465. 
[63] Andreas Weiermann. A classification of rapidly growing ramsey functions. Proc. Amer. Math. Soc., 132(2):553-561, 2004.

[64] Keita Yokoyama. On $\Pi_{1}^{1}$ conservativity of $\Pi_{2}^{1}$ theories in second order arithmetic. In C. T. Chong et al., editor, Proceedings of the 10th Asian Logic Conference, pages 375-386. World Scientific, 2009.

[65] Keita Yokoyama. Notes on the first-order part of Ramsey's theorem for pairs. In Toshio Suzuki, editor, RIMS Kokyuroku, volume 1832, pages 127-134, 2013.

[66] Keita Yokoyama. On the strength of Ramsey's theorem without $\Sigma_{1}$-induction. Math. Log. Q., 59(1-2):108-111, 2013. 